\title{
SET CONVERGENCES. AN ATTEMPT OF CLASSIFICATION
}

\author{
YVES SONNTAG AND CONSTANTIN ZĂLINESCU
}

\begin{abstract}
We endow families of nonempty closed subsets of a metric space with uniformities defined by semimetrics. Such structure is completely determined by a class (which is a family of closed sets) and a type (which is a semimetric). Two types are sufficient to define (and classify) almost all convergences known till now. These two types offer the possibility of defining other set convergences.
\end{abstract}

\section{INTRODUCTION}

The present paper develops the text of the conference [SoZ2]. Our aim is to find an adequate approach that permits

(1) to classify the notions of set convergence of closed subsets of a metric space,

(2) to search for new convergences which may satisfy certain requirements.

The second point is illustrated by two examples.

Mosco convergence, $M$, for sequences of closed convex subsets of a reflexive Banach space has excellent properties which fail to hold in general vector spaces (n.v.s.) [Be5, BBo]; is it possible to find a convergence which is similar to $M$ (in a sense that must be specified) and has good properties in n.v.s.?

The $\rho$-Hausdorff (or Attouch-Wets) convergence, $H_{\rho}$ or $A W$, has very good properties (see [AW1, 2, 3, ALW, Be5],...), but it is very strong. For instance the increasing sequence $\left(A_{n}\right)$ in $l^{2}, A_{n}=\left\{x=\left(x_{k}\right) \mid\|x\| \leq 1, x_{k}=0\right.$ for $k>n\}$ is not $H_{\rho}$-convergent. Is it possible to find a coarser convergence which retains the good properties of $H_{\rho}$ ?

We believe that our aim is achieved by considering on $\mathscr{F}(E)$-the class of nonempty closed subsets of the metric space $(E, d)$-two types $p$ and $q$, which define on $\mathscr{F}(E)$ uniformities generated by families of semimetrics. These types were suggested to us by the papers [Be2, 3, BLLN, and SoZ1] for $p$, and [Co] and the papers on $\rho$-Hausdorff convergence (loc. cit.) for $q$.

To be more specific, one gives $\varnothing \neq \mathfrak{X} \subset \mathscr{F}(E)$-which will be called class of the uniformity - and defines semimetrics

Received by the editors July $25,1991$.

1991 Mathematics Subject Classification. Primary 54A20, 54B20, 54E15; Secondary 54D55, $52 \mathrm{~A} 05$.

Key words and phrases. Convergences on hyperspace, classes of subsets, types of semimetrics, classification. 


$$
\begin{array}{lll}
\text { of type } p: & p_{X}(A, B)=|d(A, X)-d(B, X)|, & X \in \mathfrak{X}, \\
\text { of type } q: & q_{X}(A, B)=\sup _{x \in X}|d(x, A)-d(x, B)|, & X \in \mathfrak{X} .
\end{array}
$$

We get in this way for $\varnothing \neq \mathscr{A} \subset \mathscr{F}(E)$ the uniformities $(\mathscr{A}, \mathfrak{X}, p)$ (defined by $\left.\left(p_{X}\right)_{X \in \mathfrak{X}}\right)$ and $(\mathscr{A}, \mathfrak{X}, q)$ (defined by $\left.\left(q_{X}\right)_{X \in \mathfrak{X}}\right)$. These uniformities induce topologies on $\mathscr{A}$ and convergence notions, denoted $\mathfrak{X}(p), \mathfrak{X}(q)$ for nets of $\mathscr{A}$.

It is astonishing that only these two types and natural classes are sufficient to obtain and classify most part of known set convergences.

So, we shall see that the convergence $W$ of Wijsman [Wi] is defined by both types $p$ and $q$ and the class $\mathscr{S}(E)$ of singletons or the class of compact subsets $\mathscr{K}(E)$, while in n.v.s. by $p$ and the class of closed balls, too. The Hausdorff convergence $H$ and $\rho$-Hausdorff convergence are of type $q$ and classes $\{E\}$ and $\mathscr{B}(E)$ (bounded sets), respectively. The convergence $Z$ in the sense of Fisher [Fil] is of type $p$ and class $\mathscr{F}(E)$.

If $E$ is a n.v.s. the scalar convergence $S$ and the linear convergence $L$ (introduced independently by Beer [Be3] and Hess [He]) are of type $p$ and classes of hyperplanes, $\mathscr{H}(E)$, and convex sets, $\mathscr{C}(E)$, respectively. The convergence $Z_{\rho}$, introduced recently in $[\mathrm{Sh}, \mathrm{SP}, \mathrm{AAB}, \mathrm{BLu}]$ is of type $p$ and class $\mathscr{B}(E)$.

When $E$ is a reflexive Banach space, the Mosco convergence is (on $\mathscr{A}=$ $\mathscr{C}(E)$ ) of type $p$ and has as class the weak-compact sets [Be2] (or weak-compact and convex sets $[\mathrm{BP}])$, etc.

The convergence in the sense of Kuratowski [Kur] does not enter in our classification, nor does a convergence from [LSW, Lu2]. But we are led naturally to consider new notions of convergence, particularly of type $p$, which seem to be interesting (classes of weak-closed subsets, weak-closed and bounded subsets, affine closed manifolds). So we can propose three possible substitutes for $M$ and two for $H_{\rho}$.

The type $q$ is related to classical results (see [Bou3]), but $p$ is new except for the class $\mathscr{S}(E)$ [LeL].

We study different problems throughout this paper: induced topology, completeness, continuity properties of operations.

Although only two types are sufficient to describe most classical convergences, it would be interesting to introduce new types to obtain other convergences or classical topologies whose corresponding convergences were not studied, like Vietoris topology. The papers [BLLN, BLu] are, mutatis mutandis, source of interesting uniformities.

The approach presented here, by the aid of semimetrics, has some advantages

(i) comparing convergences (except for $M$ ) is done by comparing the corresponding classes;

(ii) the topologies associated to the convergences are always completely regular $\left(T_{2}\right.$ separation being natural); the semimetric structure gives the possibility of considering completion;

(iii) all the convergences obtained by this approach are adequate for obtaining estimations (quantitative aspect) due to semimetrics;

(iv) the theoretical role-which does not correspond, necessarily, to the practical role-of some convergences is made evident by the extremality of the corresponding classes (see the figure in $\S 10$ ).

Note that this article does not suppose the knowledge of the classical notions of convergence discussed here; they are defined when first met. Moreover the 
reader may consult the following books [Kur, At, Ma, KT], the papers [BaP, BLLN, FLL, SaW], and monograph in preparation [So2].

Acknowledgments. The paper was completed while the second author was visiting the Department of Mathematics of the University of Provence. Typing of the manuscript was assumed by C.N.R.S. (France) Laboratory U.R.A. 225.

\section{TERMINOLOGY AND NOTATIONS}

Let $E$ be a nonempty set and $\varnothing \neq \mathscr{A} \subset 2^{E}$. By convergence on $\mathscr{A}$ we mean that a structure of $\mathscr{L}^{*}$-space is given on $\mathscr{A}$ in the sense of [Kur, Ber]. We say that a convergence on $\mathscr{A}$ is topological if it satisfies the Moore-Smith axioms (see [Ke, p. 74]). There is a 1-1 correspondence between topological convergences and topologies on $\mathscr{A}[\mathrm{Ke}]$.

We write $\left(x_{i}\right)_{i \in K} \subset\left(A_{i}\right)_{i \in I}$ when $K \subset I$ and $x_{i} \in A_{i}$ for $i \in K$ and $\left(x_{i}\right)_{i \in I} \subset A$ when $x_{i} \in A$ for $i \in I$.

Let now $(E, d)$ be a metric space. The class of nonempty closed subsets of $E$ is denoted by $\mathscr{F}(E)$, while $\mathscr{B}(E)\left(\mathscr{K}(E), \mathscr{P}_{f}(E), \mathscr{S}(E)\right)$ denotes the class of those elements of $\mathscr{F}(E)$ which are bounded (compact, finite, singleton). When there is no danger of confusion, we write simply $\mathscr{F}, \mathscr{B}, \mathscr{K}$, etc. For $\varnothing \neq X, Y \subset E, u \in E, d(u, X)=\inf \{d(u, x) \mid x \in X\}, d(X, Y)=$ $\inf \{d(x, Y) \mid x \in X\}, e(X, Y)=\sup \{d(x, Y) \mid x \in X\}$. Also for $\varnothing \neq X \subset E$ and $\rho>0, B(X, \rho)=\{u \in E \mid d(u, X)<\rho\}, \bar{B}(X, \rho)=\{u \in E \mid d(u, X) \leq$ $\rho\}, S(X, \rho)=\{u \in E \mid d(u, X)=\rho\}$ and for $u \in E, B(u, \rho)=B(\{u\}, \rho)$ and similarly for $\bar{B}(u, \rho)$ and $S(u, \rho)$. Moreover, for $\varnothing \neq \mathfrak{X} \subset \mathscr{F}(E), u \in E$ and $R>0 \quad \mathfrak{X}_{u, R}=\{X \in \mathfrak{X} \mid X \subset \bar{B}(u, R)\}$.

Suppose now that $(E,\|\cdot\|)$ is a real normed vector space (abbreviated n.v.s.). For $u, v \in E$, the segment $[u, v]$ is $\{\lambda u+(1-\lambda) v \mid \lambda \in[0,1]\}$, while for $\varnothing \neq$ $A, B \subset E$ and $\lambda \in \mathbf{R}, A+B=\{a+b \mid a \in A, b \in B\}, \lambda A=\{\lambda a \mid a \in A\}$. In this case one takes $U=\bar{B}(0,1)$ and $\mathfrak{X}_{R}=\mathfrak{X}_{0, R}$. Also, for $\varnothing \neq A \subset E \operatorname{conv}(A)$ $(\overline{c o n v}(A), \operatorname{span}(A))$ denotes the convex hull (closed convex hull, linear hull) of $A$.

The class of nonempty closed convex subsets of $E$ is denoted by $\mathscr{C}(E)$ while $\mathscr{V}(E)$ denotes the class of affine closed subsets of $E ; \mathscr{V}_{f}(E) \quad\left(\mathscr{V}_{c}(E), \mathscr{H}(E)\right)$ denotes the class of those elements of $\mathscr{V}(E)$ of finite dimension (finite codimension, codimension 1).

The topological dual of $(E,\|\cdot\|)$ is denoted $\left(E^{\prime},\|\cdot\|^{\prime}\right)$, where $\|\cdot\|^{\prime}$ is the corresponding dual norm. For $\varphi \in E^{\prime},\langle u, \varphi\rangle=\varphi(u)$. The weak topology of $E\left(w^{*}\right.$ topology of $\left.E^{\prime}\right)$ is denoted by $\sigma\left(E, E^{\prime}\right)\left(\sigma\left(E^{\prime}, E\right)\right)$.

So $\mathscr{F}_{\sigma}(E)\left(\mathscr{K}_{\sigma}(E)\right)$ is the class of $\sigma\left(E, E^{\prime}\right)$-closed (-compact) subsets of $E$.

Also, for $\varnothing \neq A \subset E$ and $\varphi \in E^{\prime}, s_{A}(\varphi)=\sup \{\varphi(a) \mid a \in A\}$ (i.e. $s_{A}$ is the support function of $A$ ).

In $E=l^{2}, e_{n}=(0, \ldots, 0,1,0, \ldots)$, where 1 is at $n$th place, $E_{n}=$ $\operatorname{span}\left\{e_{1}, \ldots, e_{n}\right\}$ and $P_{n}$ is the orthogonal projection of $E$ onto $E_{n}$. The notations $e_{n}, E_{n}$ are the same for $l^{p} \quad(1 \leq p \leq \infty)$.

\section{Classes and types. Elementary PROPerties}

Let $(E, d)$ be a metric space and $\varnothing \neq \mathscr{A} \subset \mathscr{F}(E)$. We intend to endow $\mathscr{A}$ with a uniformity. Typically $\mathscr{A}$ is $\mathscr{F}(E), \mathscr{B}(E), \mathscr{K}(E)$ or $\mathscr{F}(E)_{u, R}$, while for $E$ a n.v.s. $\mathscr{A}$ may be also $\mathscr{C}(E), \mathscr{C}(E) \cap \mathscr{B}(E), \mathscr{F}_{\sigma}(E)$, etc. 
Let $\varnothing \neq \mathfrak{X} \subset \mathscr{F}(E)$ be another family. $\mathfrak{X}$ is called the class of the uniformity or of the convergence. This family will represent the index set for the semimetrics on $\mathscr{A}$ which will define the uniformity. Let us note that we do not need to have $\mathfrak{X} \subset \mathscr{A}$ or $\mathscr{A} \subset \mathfrak{X}$.

The type $p$. Let $\mathscr{A}$ and $\mathfrak{X}$ be given. For $X \in \mathfrak{X}$ we may consider the function $f_{X}: \mathscr{A} \rightarrow \mathbf{R}, f_{X}(A)=d(A, X)$. The coarser uniformity on $\mathscr{A}$ for which every $f_{X}, X \in \mathfrak{X}$ is uniformly continuous may be defined by the semimetrics (see [Bou2, §1, no. 2]):

$$
p_{X}(A, B)=\left|f_{X}(A)-f_{X}(B)\right|=|d(A, X)-d(B, X)|, \quad X \in \mathfrak{X} .
$$

So $\mathscr{A}$ is endowed with a uniformity characterized by the class $\mathfrak{X}$ and the type $p$ corresponding to the semimetrics $\left(p_{X}\right)_{X \in \mathfrak{X}}$. We denote by $(\mathscr{A}, \mathfrak{X}, p)$ this uniformity abbreviated $\mathfrak{X}(p)$ when $\mathscr{A}$ is already specified. The topology determined by $(\mathscr{A}, \mathfrak{X}, p)$ is the initial (weak) topology on $\mathscr{A}$ (see [Bou1, $\S 2$, no. 3]) defined by the family $\left(f_{X}\right)_{X \in X}$; it is the coarsest topology on $\mathscr{A}$ for which $f_{X}$ is continuous for all $X \in \mathfrak{X}$.

Let $I$ be a directed set (see [Kö, Ke]), $\left(A_{i}\right)_{i \in I} \subset \mathscr{A}$ a net and $A \in \mathscr{A}$. The convergence associated to $\mathfrak{X}(p)$ is defined by

$$
A=\mathfrak{X}(p)-\lim \left(A_{i}\right) \quad \text { iff } \quad \lim d\left(A_{i}, X\right)=d(A, X) \text { for all } X \in \mathfrak{X} .
$$

The type $q$. Let $C(E, \mathbf{R})$ be the class of continuous functions from $(E, d)$ to $\mathbf{R}$ and let $\varnothing \neq \mathfrak{X} \subset \mathscr{F}(E)$. We may consider on $C(E, \mathbf{R})$ the uniformity called the uniformity of the uniform convergence on every $X \in \mathfrak{X}$ (see [Bou3] for a detailed study of this uniformity). This uniformity may be defined by the semimetrics (taking also the value $\infty$ )

$$
d_{X}(f, g)=\sup _{x \in X}|f(x)-g(x)|, \quad X \in \mathfrak{X} .
$$

One may also consider (see [Co]) the map $j: \mathscr{A} \rightarrow C(E, \mathbf{R})$ with $j(A): E \rightarrow \mathbf{R}$, $j(A)(u)=d(u, A)$. This map is injective (because $d(u, A)=d(u, B)$ for all $u \in E$ iff $\operatorname{cl}(A)=\operatorname{cl}(B)$ iff $A=B$ because $\mathscr{A} \subset \mathscr{F}(E))$. Let $\Delta(\mathscr{A})=$ $\{j(A) \mid A \in \mathscr{A}\} \subset C(E, \mathbf{R})$. It is natural to consider on $\Delta(\mathscr{A})$ the trace of the above uniformity of $C(E, \mathbf{R})$. In this way, we obtain a uniformity on $\mathscr{A}$ determined by the semimetrics

$$
q_{X}(A, B)=d_{X}(j(A), j(B))=\sup _{x \in X}|d(x, A)-d(x, B)|, \quad X \in \mathfrak{X} .
$$

So $\mathscr{A}$ is endowed with a uniformity, denoted $(\mathscr{A}, \mathfrak{X}, q)$ or simply $\mathfrak{X}(q)$, characterized by the class $\mathfrak{X}$ and the type $q$ corresponding to the family of semimetrics $\left(q_{X}\right)_{X \in \mathfrak{X}}$.

Thus the topology determined by $(\mathscr{A}, \mathfrak{X}, q)$ on $\mathscr{A}$ is the topology of uniform convergence on $X, X \in \mathfrak{X}$, of the functions $d(\cdot, A), A \in \mathscr{A}$.

Let $\left(A_{i}\right)_{i \in I} \subset \mathscr{A}$ be a net and $A \in \mathscr{A}$. The convergence associated to $\mathfrak{X}(q)$ is defined by

$$
A=\mathfrak{X}(q)-\lim \left(A_{i}\right) \quad \text { iff } \quad \lim \left(\sup _{x \in X}\left|d\left(x, A_{i}\right)-d(x, A)\right|\right)=0 \quad \text { for all } X \in \mathfrak{X} .
$$

Remark. The notations $A=\mathfrak{X}(p)-\lim \left(A_{i}\right)$ and $A=\mathfrak{X}(q)-\lim \left(A_{i}\right)$ presuppose unicity for $A$. If there is no unicity, = must be replaced by $\epsilon$. 
Remark. In the literature there are convergences defined only for small (enough) families, like $\mathscr{C}(E)$. For types $p$ and $q$ we always may extend them to $\mathscr{F}(E)$.

Remark. The types $p$ and $q$ may also be defined in semimetric space $\left(E_{i}\right.$, $\left.\left(d_{i}\right)_{i \in I}\right)$. Taking the families $\left\{p_{X, i} \mid X \in \mathfrak{X}, i \in I\right\}$ and $\left\{q_{X, i} \mid X \in \mathfrak{X}, i \in I\right\}$ respectively, where $p_{X, i}$ is $p_{X}$ corresponding to $d_{i}$. In this case one may also take

$$
\begin{array}{ll}
p_{X}(A, B)=\sup _{i \in I}\left|d_{i}(A, X)-d_{i}(B, X)\right|, & X \in \mathfrak{X}, \\
q_{X}(A, B)=\sup _{i \in I} \sup _{x \in X}\left|d_{i}(x, A)-d_{i}(x, B)\right|, & X \in \mathfrak{X} .
\end{array}
$$

For instance if $(E, d)$ is a metric space $\left\{\left(d_{i}\right)_{i} \in I\right\}$ may be the set of all metrics on $E$ defining the same topology (or the same uniformity, or the same bounded sets, etc.) as $d$. See [BLLN, BLu] for potential applications.

\section{ELEMENTARY PROPERTIES}

1. It is easy to show that $p_{X}(A, B) \leq q_{X}(A, B)$ for all $A, B, X \in \mathscr{F}(E)$. So for $\mathscr{A}$ and $\mathfrak{X}$ fixed the uniformity $(\mathscr{A}, \mathfrak{X}, q)$ (and the corresponding topology and convergence) is finer than the uniformity $(\mathscr{A}, \mathfrak{X}, p)$.

2. If $\mathfrak{X}$ and $\mathscr{Y}$ are such that $\mathfrak{X} \supset \mathscr{Y} \neq \varnothing$ one has that $(\mathscr{A}, \mathfrak{X}, p)$ is finer than $(\mathscr{A}, \mathscr{Y}, p)($ and $(\mathscr{A}, \mathfrak{X}, q)$ is finer than $(\mathscr{A}, \mathscr{Y}, q))$.

3. If $\mathfrak{X}, \mathscr{Y} \neq \varnothing$ and for each $Y \in \mathscr{Y}$ there exists $X \in \mathfrak{X}$ such that $Y \subset X$ then $(\mathscr{A}, \mathfrak{X}, q)$ is finer than $(\mathscr{A}, \mathscr{Y}, q)$.

4. If $\mathfrak{X} \supset \mathscr{S}(E)$ then $(\mathscr{A}, \mathfrak{X}, p)$ is $\left(T_{2}\right.$-) separated. Indeed, if $p_{X}(A, B)=$ 0 for all $X \in \mathfrak{X}$ then $d(x, A)=d(x, B)$ for all $x \in E$ and so $A=B$. So, in this case we have the uniqueness of the limit (when it exists). An example of nonseparated uniformity is $(\mathscr{A},\{E\}, p)$.

5. If $\bigcup\{X \mid X \in \mathfrak{X}\}=E$ then $(\mathscr{A}, \mathfrak{X}, q)$ is separated.

The condition $\bigcup\{X \mid X \in \mathfrak{X}\}=E$ is verified by each family introduced in $\S 2$, while the condition $\mathfrak{X} \supset \mathscr{S}(E)$ is verified by each family introduced in $\S 2$, excepting $\mathscr{H}(E)$ for $\operatorname{dim}(E) \geq 2$ and $\mathscr{V}_{c}(E)$ for $\operatorname{dim}(E)=\infty$.

Concerning these classes we have

Proposition 3.1. Let $E$ be a n.v.s. Then $(\mathscr{C}, \mathfrak{X}, p)$ is separated for $\mathfrak{X} \supset \mathscr{H}(E)$.

Proof. It is sufficient to show that $(\mathscr{C}, \mathscr{H}, p)$ is separated because $(\mathscr{C}, \mathfrak{X}, p)$ is finer. Suppose that $A, B \in \mathscr{C}$ and $A \neq B$. We may suppose that $A \not \subset B$. Then there exists $a \in A, a \notin B$. By a separation theorem there exists $\varphi \in E^{\prime}$, $\|\varphi\|^{\prime}=1$ such that $\varphi(a)>\sup \{\varphi(b) \mid b \in B\}$. Let us take $H=\{u \in E \mid \varphi(u)=$ $\varphi(a)\}$. Then $d(A, H)=0$ while

$$
\begin{aligned}
d(B, H) & =\inf \{d(b, H) \mid b \in B\}=\inf \{|\varphi(b)-\varphi(a)| \mid b \in B\} \\
& =\varphi(a)-\sup \{\varphi(b) \mid b \in B\}>0 .
\end{aligned}
$$

Therefore $p_{H}(A, B)>0$.

We utilised Ascoli's formula

$$
d(b,\{x \mid \varphi(x)=\gamma\})=|\varphi(b)-\gamma| /\|\varphi\|^{\prime} .
$$


We recall the definition of Kuratowski convergence [Kur, Ber, At, So2]. We define $\liminf \left(A_{i}\right)$ and $\lim \sup \left(A_{i}\right)$ for a net $\left(A_{i}\right)_{i \in I} \subset 2^{E} \backslash\{\varnothing\}$ :

$$
\begin{gathered}
\liminf \left(A_{i}\right)=\left\{x \in E \mid \lim d\left(x, A_{i}\right)=0\right\}, \\
\lim \sup \left(A_{i}\right)=\left\{x \in E \mid \liminf d\left(x, A_{i}\right)=0\right\} .
\end{gathered}
$$

Generally, when $\liminf \left(A_{i}\right)=\lim \sup \left(A_{i}\right)=A$ we say that $\left(A_{i}\right)$ converges in the sense of Kuratowski to $A$ and denote it by $A=K-\lim \left(A_{i}\right)$.

Let us note that we always have [Ber, At],

$$
\operatorname{cl}\left(\bigcup_{i \in I} \bigcap_{j \geq i} A_{j}\right) \subset \liminf \left(A_{i}\right) \subset \lim \sup \left(A_{i}\right)=\bigcap_{i \in I} \mathrm{cl}\left(\bigcup_{j \geq i} A_{j}\right) \text {. }
$$

From the above formula we have that $\lim \sup \left(A_{i}\right)$ is closed. The same is true for $\liminf \left(A_{i}\right)=A$. Indeed if $\bar{x} \in \operatorname{cl}(A)$ and $\varepsilon>0$ then there exists $x \in A$ such that $d(x, \bar{x})<\varepsilon / 2$; as $x \in \liminf \left(A_{i}\right)$ there exists $i_{\varepsilon}$ such that for all $i \geq i_{\varepsilon}$ we have $d\left(x, A_{i}\right)<\varepsilon / 2$ and so $d\left(\bar{x}, A_{i}\right) \leq d\left(x, A_{i}\right)+d(x, \bar{x})<\varepsilon$; therefore $\lim d\left(\bar{x}, A_{i}\right)=0$ which shows that $\bar{x} \in A$.

Also, from the above formula it follows that for $\left(A_{i}\right) \subset \mathscr{F}(E)$ increasing, i.e. $A_{i} \subset A_{j}$ for $i \leq j$,

$$
\liminf \left(A_{i}\right)=\lim \sup \left(A_{i}\right)=\operatorname{cl}\left(\bigcup_{i \in I} A_{i}\right),
$$

and for $\left(A_{i}\right) \subset \mathscr{F}(E)$ decreasing, i.e. $A_{i} \supset A_{j}$ for $i \geq j$,

$$
\liminf \left(A_{i}\right)=\limsup \left(A_{i}\right)=\bigcap_{i \in I} A_{i} .
$$

The next result is surely known but it seems that is not given explicitly elsewhere.

Lemma 3.2. Let $\left(A_{i}\right)_{i \in I}$ be a net in $\mathscr{F}(E)$. Then $u \in \lim \inf \left(A_{i}\right)$ iff there exists $\left(u_{i}\right)_{i \in I} \subset\left(A_{i}\right)_{i \in I}$ such that $\lim \left(u_{i}\right)=u$.

Proof. Suppose that $u \in \liminf \left(A_{i}\right)$. Since $A_{i}$ is closed, there exists $u_{i} \in A_{i}$ such that $d\left(u, u_{i}\right) \leq 2 d\left(u, A_{i}\right), i \in I$. Therefore $\left(u_{i}\right)_{i \in I} \subset\left(A_{i}\right)_{i \in I}$ and $\lim \left(u_{i}\right)=u$. Conversely, if there exists $\left(u_{i}\right)_{i \in I} \subset\left(A_{i}\right)_{i \in I}$ such that $\lim \left(u_{i}\right)=u$ then $d\left(u, A_{i}\right) \leq d\left(u, u_{i}\right)$ and so $u \in \liminf \left(A_{i}\right)$.

The next result will be important for the sequel (see [FrLL] for (1) $\Leftrightarrow(3)$ ).

Theorem 3.3. Let $\left(A_{i}\right)_{i \in I} \subset \mathscr{F}(E)$ be a net and $A \in \mathscr{F}(E)$. The following assertions are equivalent:

(1) $A \subset \liminf \left(A_{i}\right)$,

(2) $d(A, X) \geq \limsup d\left(A_{i}, X\right)$ for all $X \in \mathscr{F}(E)$,

(3) $d(u, A) \geq \limsup d\left(u, A_{i}\right)$ for all $u \in E$,

(4) $\mu(f, A) \geq \lim \sup \mu\left(f, A_{i}\right)$ for every upper semicontinuous function $f: E \rightarrow \mathbf{R}$,

(5) $\mu(f, A) \geq \lim \sup \mu\left(f, A_{i}\right)$ for every lipschitzian function $f: E \rightarrow \mathbf{R}$, where $\mu(f, A)=\inf \{f(x) \mid x \in A\}$.

Proof. (1) $\Rightarrow(4)$. Indeed, let $f: E \rightarrow \mathbf{R}$ be upper semicontinuous (u.s.c.) and $u \in A$. Then there exists, by Lemma 3.2, $\left(u_{i}\right)_{i \in I} \subset\left(A_{i}\right)_{i \in I}$ such that $\lim \left(u_{i}\right)=$ 
$u$. As $f$ is u.s.c. we have $\lim \sup \mu\left(f, A_{i}\right) \leq \limsup f\left(u_{i}\right) \leq f(u)$. As $u \in A$ is arbitrary, it follows that $\lim \sup \mu\left(f, A_{i}\right) \leq \mu(f, A)$. (4) $\Rightarrow(5)$ is obvious. To obtain $(5) \Rightarrow(2)$ take $X \in \mathscr{F}(E)$ and $f=d(\cdot, X)$. The conclusion follows from the well-known inequality $|d(x, X)-d(y, X)| \leq d(x, y)$ for $x, y \in E$. Taking $X=\{u\}$, we obtain $(2) \Rightarrow(3)$, while $(3) \Rightarrow(1)$ is immediate (take $u \in A)$.

Proposition 3.4. Let $\left(A_{i}\right)_{i \in I} \subset \mathscr{F}(E), A \in \mathscr{F}(E)$, and $\varnothing \neq \mathfrak{X} \subset \mathscr{F}(E)$.

(1) If $A_{i} \subset A$ for all $i \in I$ and $A \subset \liminf \left(A_{i}\right)$ then $A=\mathfrak{X}(p)-\lim \left(A_{i}\right)$.

(2) If $A_{i} \subset A_{j}$ for $i \leq j$ then $\mathfrak{X}(p)-\lim \left(A_{i}\right)=\operatorname{cl}\left(\bigcup_{i \in I} A_{i}\right)$.

(3) If $\mathscr{S}(E) \subset \mathfrak{X}$ and $A=\mathfrak{X}(p)-\lim \left(A_{i}\right)$ then $A=K-\lim \left(A_{i}\right)$.

Proof. (1) By using Theorem 3.3, from $A \subset \liminf \left(A_{i}\right)$ we get $d(A, X) \geq$ $\limsup d\left(A_{i}, X\right)$ for all $X \in \mathfrak{X}$. As $A \supset A_{i}, d(A, X) \leq d\left(A_{i}, X\right)$ for all $i$ and for all $X \in \mathfrak{X}$, and so $d(A, X) \leq \liminf d\left(A_{i}, X\right)$. Therefore $d(A, X)=$ $\lim d\left(A_{i}, X\right)$ for all $X \in \mathfrak{X}$ and so $\bar{A}=\mathfrak{X}(p)-\lim \left(A_{i}\right)$.

(2) Let $A=\operatorname{cl}\left(\bigcup_{i \in I} A_{i}\right): A \supset A_{i}$ for $i \in I$. We saw above that in this case $A$ equals $\liminf \left(A_{i}\right)$. Thus, by (1), $\mathfrak{X}(p)-\lim \left(A_{i}\right)=A$.

(3) As $A=\mathfrak{X}(p)-\lim \left(A_{i}\right)$ and $\mathscr{S}(E) \subset \mathfrak{X}$ we have $d(u, A)=\lim d\left(u, A_{i}\right)$ for all $u \in E$. Hence, for $u \in A, \lim d\left(u, A_{i}\right)=d(u, A)=0$ and so $u \in \liminf \left(A_{i}\right)$. On the other hand, for $u \in \limsup \left(A_{i}\right), \lim \inf d\left(u, A_{i}\right)=$ $\lim d\left(u, A_{i}\right)=0=d(u, A)$ and so $u \in A$. Thus we get $A=\liminf \left(A_{i}\right)=$ $\lim \sup \left(A_{i}\right)$, i.e. $A=K-\lim \left(A_{i}\right)$.

Remark. Let $\left(A_{i}\right)_{i \in I} \subset \mathscr{A}$ be increasing and $\mathscr{S}(E) \subset \mathfrak{X}$. Then $\left(A_{i}\right)_{i \in I}$ is $\mathfrak{X}(p)$-convergent iff $\operatorname{cl}\left(\bigcup_{i \in I} A_{i}\right) \in \mathscr{A}$.

Indeed, on the one hand, if the limit exists then it is unique. On the other hand, if the limit exists in $(\mathscr{A}, \mathfrak{X}, p)$ then it exists in $\mathscr{F}(E), \mathfrak{X}, p)$ and is $\operatorname{cl}\left(\bigcup_{i \in I} A_{i}\right)$.

\section{Convergences Related to TYPE $q$}

Wijsman convergence, $W$. Let $(E, d)$ be a metric space.

Definition 4.1. The net $\left(A_{i}\right)_{i \in I} \subset \mathscr{F}(E)$ converges to $A \in \mathscr{F}(E)$ in the sense of Wijsman, denoted $A=W-\lim \left(A_{i}\right)$, if

$$
\lim d\left(u, A_{i}\right)=d(u, A) \text { for all } u \in E .
$$

We mention some references related to $W$ : [Wi, BaP, FrLL, LeL, So2, BLLN, Be6].

From the definition we see that $A=W-\lim \left(A_{i}\right)$ iff $A=\mathscr{S}(p)-\lim \left(A_{i}\right)$ iff $A=\mathscr{S}(q)-\lim \left(A_{i}\right)$.

Using eventually Theorem 3.3 , we have that $A=K-\lim \left(A_{i}\right)$ if $A=W-$ $\lim \left(A_{i}\right)$, and therefore $A=\bigcap_{i \in I} \operatorname{cl}\left(\bigcap_{j \geq 1} A_{j}\right)$ when $A=W-\lim \left(A_{i}\right)$.

The next result shows that Wijsman convergence is closer to type $q$.

Proposition 4.2. The uniformities $(\mathscr{F}(E), \mathscr{S}, q),(\mathscr{F}(E), \mathfrak{X}, p)$ and $(\mathscr{F}(E)$, $\mathfrak{X}, q)$ are equivalent for every $\mathfrak{X}$ such that $\mathscr{S}=\mathscr{S}(E) \subset \mathfrak{X} \subset \mathscr{K}(E)$.

Proof. As for $\mathscr{S} \subset \mathfrak{X} \subset \mathscr{K}$ we have $(\mathscr{F}(E), \mathscr{S}, q)=(\mathscr{F}(E), \mathscr{S}, p) \subset$ $(\mathscr{F}(E), \mathfrak{X}, p) \subset(\mathscr{F}(E), \mathfrak{X}, q) \subset(\mathscr{F}(E), \mathscr{K}, q)$, it is sufficient to show that $(\mathscr{F}(E), \mathscr{S}, q)$ is finer than $(\mathscr{F}(E), \mathscr{K}, q)$. For this aim let $X \in \mathscr{K}$ and 
$\varepsilon>0$. We must show that there exist $x_{1}, x_{2}, \ldots, x_{n} \in E$ and $\varepsilon^{\prime}>0$ such that $\left\{(A, B)|| d\left(x_{i}, A\right)-d\left(x_{i}, B\right) \mid<\varepsilon^{\prime}, 1 \leq i \leq n\right\} \subset\left\{(A, B) \mid q_{X}(A, B)<\varepsilon\right\}$.

Indeed, as $X$ is compact, there exist $x_{1}, x_{2}, \ldots, x_{n} \in X$ such that $X \subset$ $\bigcup_{i=1}^{n} B\left(x_{i}, \varepsilon / 3\right)$. Let $(A, B) \in \mathscr{F}(E) \times \mathscr{F}(E)$ such that $\left|d\left(x_{i}, A\right)-d\left(x_{i}, B\right)\right|<$ $\varepsilon / 3=\varepsilon^{\prime}$ for all $i, 1 \leq i \leq n$. Let us consider $x \in X$; then there exists $i$ such that $x \in B\left(x_{i}, \varepsilon^{\prime}\right)$. Thus, $|d(x, A)-d(x, B)| \leq\left|d(x, A)-d\left(x_{i}, A\right)\right|+$ $\left|d\left(x_{i}, A\right)-d\left(x_{i}, B\right)\right|+\left|d\left(x_{i}, B\right)-d(x, B)\right| \leq d\left(x, x_{i}\right)+\varepsilon^{\prime}+d\left(x_{i}, x\right)<3 \varepsilon^{\prime}=\varepsilon$. Therefore, $q_{X}(A, B)=\max _{x \in X}|d(x, A)-d(x, B)|<\varepsilon$.

The use of the semimetrics $p_{\{u\}}=q_{\{u\}}, u \in E$, for defining $W$ is not new: see $[\mathrm{Co}, \mathrm{LeL}]$ and others.

A natural question is: may $W$ be described by $\mathfrak{X}(p)$ when $\mathfrak{X}$ contains noncompact sets. The answer is affirmative, at least when $E$ is a normed space as we shall see later on.

Hausdorff convergence, $H$. For $X, Y \in \mathscr{F}(E)$ let

$$
h(X, Y)=\max \{e(X, Y), e(Y, X)\} \in \overline{\mathbf{R}} .
$$

It is known that $h$ is an extended real valued metric.

Definition 4.3. The net $\left(A_{i}\right)_{i \in I} \subset \mathscr{F}(E)$ converges to $A \in \mathscr{F}(E)$ in the sense of Hausdorff, denoted $A=H-\lim \left(A_{i}\right)$, if: $\lim h\left(A_{i}, A\right)=0$.

We mention some references related to $H$ : [Kur, Ma, KT, So2] etc. It is known, for a long time (see [Kur, Co]), that

$$
h(A, B)=\sup _{u \in E}|d(u, A)-d(u, B)| .
$$

This shows that in $\mathscr{F}(E)$, for all $\mathfrak{X} \subset \mathscr{F}(E)$ such that $E \in \mathfrak{X}$, we have: $A=H-\lim \left(A_{i}\right)$ iff $A=\mathfrak{X}(q)-\lim \left(A_{i}\right)$.

Thus, if $E$ is a n.v.s. then the classes $\mathscr{C}, \mathscr{V}, \mathscr{V}_{c}, \mathscr{F}_{\sigma}$ define on $\mathscr{F}(E)$ the same uniformity for type $q$, the corresponding convergence being $H$.

Note that $A=H-\lim \left(A_{i}\right)$ iff the net of functions $\left(d\left(\cdot, A_{i}\right)\right)_{i \in I}$ converges uniformly to $d(\cdot, A)$ on $E$ while $A=W-\lim \left(A_{i}\right)$ iff the same net of functions converges pointwise to $d(\cdot, A)$. Thus it is reasonable to consider intermediate convergences. Strangely enough, the first such convergence was explicitly considered in 1987.

$\rho$-Hausdorff or Attouch-Wets convergence, $H_{\rho}$ or $A W$. Let us fix $\bar{u} \in E$ and for $\rho>0$ let us denote $A_{\rho}=A \cap \bar{B}(\bar{u}, \rho)$. For $A, B \in \mathscr{F}(E)$ let us take $e_{\rho}(A, B)=e\left(A_{\rho}, B\right)$ and $h_{\rho}(A, B)=\max \left\{e_{\rho}(A, B), e_{\rho}(B, A)\right\}$. Because $A_{\rho}$ may be empty we consider that $e(\varnothing, X)=0$ for all $X \subset E$.

Definition 4.4. The net $\left(A_{i}\right)_{i \in I} \subset \mathscr{F}(E)$ converges to $A \in \mathscr{F}(E)$ in the sense $\rho$-Hausdorff or Attouch-Wets if for all $\rho>0, \lim h_{\rho}\left(A_{i}, A\right)=0$.

Although this convergence is so young the references concerning it are numerous: [Mo1, So2, Be4, 5, AW1, 2, 3, ALW, AZ1, 2, AP1, 2, Pe2, Lu1, 2, SP, $\mathrm{Sh}$, etc.

Note that $h_{\rho}$ is not a semimetric (it does not verify the triangle inequality). Remark also that the convergence $H_{\rho}$ does not depend on $\bar{u}$ (see [Az1]). 
Just recently [Be4, Az1, AW2, 3, AP2, $\mathrm{CoP}]$ it was observed that in $\mathscr{F}(E)$, $H_{\rho}-\lim \left(A_{i}\right)=A$ if and only if

$$
\lim \left(\sup _{x \in X}\left|d\left(x, A_{i}\right)-d(x, A)\right|\right)=0 \text { for all } X \in \mathscr{B}(E),
$$

i.e. in $\mathscr{F}(E)$,

$$
A=H_{\rho}-\lim \left(A_{i}\right) \quad \text { iff } \quad A=\mathscr{B}(q)-\lim \left(A_{i}\right) .
$$

Note that the uniformity $(\mathscr{F}, \mathscr{B}, q)$ is obtained also replacing $\mathscr{B}$ by $\mathfrak{X}=$ $\{\bar{B}(\bar{u}, \rho) \mid \rho>0\}$ or $\mathfrak{X}=\{\bar{B}(u, \rho) \mid u \in E, \rho>0\}$, while for $E$ a n.v.s. the same uniformity is obtained for $\mathfrak{X}=\mathscr{C} \cap \mathscr{B}$ and $\mathfrak{X}=\mathscr{F}_{\sigma} \cap \mathscr{B}$.

Remark. G. Salinetti and R. Wets in [SaW] have introduced a different type of convergence:

$$
A=H_{\rho \rho}-\lim \left(A_{i}\right) \quad \text { iff } \exists \rho_{0}>0, \forall \rho \geq \rho_{0}: \lim h\left(\left(A_{i}\right)_{\rho}, A_{\rho}\right)=0 .
$$

For $E$ a n.v.s., on $\mathscr{C}(E)$ this convergence coincides with $H_{\rho}$ (see [Az1 and AW3]).

The convergence $H_{\rho \rho}$ is not included in our classification. One must probably introduce new types such as $p_{X, Y}(A, B)=p_{X}(A \cap Y, B \cap Y), q_{X, Y}(A, B)=$ $q_{X}(A \cap Y, B \cap Y)$, with $X \in \mathfrak{X}, Y \in \mathscr{Y}$.

While on this subject, in $[\mathrm{SaW}]$ it is conjectured: Suppose that $A_{n}, n \in \mathbf{N}$, are nonempty closed connected subsets of $E$ (a finite dimensional space). Then $A=K-\lim \left(A_{n}\right)$ iff there exists $\sigma_{0}>0$ such that for $\sigma>\sigma_{0}$,

$$
\lim _{\substack{n \rightarrow \infty \\ \rho \downarrow \sigma}} h\left(\left(A_{n}\right)_{\rho}, A_{\rho}\right)=0 .
$$

The answer of this conjecture is negative.

Example 4.5. Let $E=\mathbf{R}^{2}$ be endowed with the Euclidean norm and consider

$$
\begin{aligned}
A_{n}^{p}= & \left(\left(\left\{2^{p}\right\} \cup\left\{2^{p}+\frac{1}{k} \mid k>n\right\}\right) \times\left[2^{p}+1, \infty\right)\right) \\
& \cup\left(\left\{2^{p}+\frac{1}{k} \mid 1 \leq k \leq n\right\} \times[0, \infty)\right), \\
A_{n}= & \bigcup_{p=1}^{\infty} A_{n}^{p} \cup\{(x, 2 x) \mid x \geq 0\} \quad \text { for } n \geq 1, \\
A^{p}= & \left(\left\{2^{p}\right\} \cup\left\{2^{p}+\frac{1}{k} \mid k \geq 1\right\}\right) \times[0, \infty), \\
A= & \bigcup_{p=1}^{\infty} A^{p} \cup\{(x, 2 x) \mid x \geq 0\} .
\end{aligned}
$$

We have that $A_{n}$ is connected and closed for every $n, A=K-\lim \left(A_{n}\right)$, but for every $p \in \mathbf{N}, p \geq 2$,

$$
\lim _{\substack{n \rightarrow \infty \\ s \downarrow 2^{p}}} e\left(A_{s},\left(A_{n}\right)_{s}\right) \neq 0 .
$$

Indeed, for $p \in \mathbf{N} \backslash\{0,1\}$ there exists $\varepsilon=\frac{1}{2}$ such that $\forall \delta>0, \quad \forall q \in \mathbf{N}^{*}, \exists s \in\left(2^{p}, 2^{p}+\delta\right), \quad \exists n \geq q: A_{s} \not \subset\left(A_{n}\right)_{s}+\bar{B}(0, \varepsilon)$. 
Let $\delta>0$ and $q \in \mathbf{N}$ be fixed and take $n=q+1,2^{p}<s<\min \left\{\delta, \frac{1}{n+1}\right\}+2^{p} \leq$ $2^{p}+\frac{1}{n+1}<2^{p}+1$. Then $\left(A_{n}\right)_{s}=\left[\left(A_{n}^{1} \cup A_{n}^{2} \cup \cdots \cup A_{n}^{p-1}\right) \cap \bar{B}(0, s)\right] \cup[\{(x, 2 x) \mid x \geq$ $0\} \cap \bar{B}(0, s)]$. It is obvious that $\left(2^{p}, 0\right) \in A_{s}$ but $\left(2^{p}, 0\right) \notin\left(A_{n}\right)_{s}+\bar{B}(0, \varepsilon)$.

In a general n.v.s. $E$, we have

$$
\begin{aligned}
\left(\mathscr{F}, \mathscr{K}_{\sigma} \cap \mathscr{C}, q\right) & \subset\left(\mathscr{F}, \mathscr{K}_{\sigma}, q\right) \subset\left(\mathscr{F}, \mathscr{F}_{\sigma} \cap \mathscr{B}, q\right)= \\
& \subset(\mathscr{F}, \mathscr{C} \cap \mathscr{B}, q)=(\mathscr{F}, \mathscr{B}, q) .
\end{aligned}
$$

Using the theorem of Krein (or Krein-Smulian) (see [DS, p. 434]) for $K \in \mathscr{K}_{\sigma}$ we have $\overline{\operatorname{conv}}(K) \in \mathscr{K}_{\sigma} \cap \mathscr{C}$. So, by property 3 of $\S 3,\left(\mathscr{F}, \mathscr{K}_{\sigma}, q\right)=\left(\mathscr{F}, \mathscr{K}_{\sigma} \cap\right.$ $\mathscr{C}, q)$. Note that in a reflexive Banach space all the above five uniformities coincide.

When $E$ is a nonreflexive Banach space the topology defined by $(\mathscr{F}, \mathscr{B}, q)$ may be strictly finer than that defined by $\left(\mathscr{F}, \mathscr{K}_{\sigma}, q\right)$.

Example 4.6. Let $E=l^{1}, A_{n}=\left[0, e_{n}\right]$ for $n \in \mathbf{N}^{*}$ and $A=\{0\}$. Then $\mathscr{K}_{\sigma}(q)-\lim \left(A_{n}\right)=\mathscr{K}(q)-\lim \left(A_{n}\right)=W-\lim \left(A_{n}\right)$ but $\mathscr{B}(q)-\lim \left(A_{n}\right)$ does not exist.

Indeed $d(u, A)=\|u\|$ and for $u=\left(\xi_{1}, \ldots, \xi_{n}, \ldots\right), d\left(u, A_{n}\right)=$ $\inf _{\lambda \in[0,1]}\left\|u-\lambda e_{n}\right\|=\|u\|-\left|\xi_{n}\right|+\inf _{\lambda \in[0,1]}\left|\xi_{n}-\lambda\right|=\|u\|-\left|\xi_{n}\right|+$ $\max \left\{-\xi_{n}, 0\right\}$ for $n \geq n_{0}\left(\lim \left(\xi_{n}\right)=0\right)$.

Therefore $W-\lim \left(A_{n}\right)=A$. As $\mathscr{K}=\mathscr{K}_{\sigma}$ in $l^{1}$ (see [Kö, p. 281]), by Proposition 4.2, we have $\mathscr{K}_{\sigma}-\lim \left(A_{n}\right)=A$. Let us take $X=S(0,1) \in \mathscr{B}$; then $\sup _{x \in X}\left|d\left(x, A_{n}\right)-d(x, A)\right| \geq\left|d\left(e_{n}, A\right)-d\left(e_{n}, A_{n}\right)\right|=1$ and so $\left(A_{n}\right)$ is not $\mathscr{B}(q)$-convergent.

The convergence $\mathscr{K}_{\sigma}(q)$ is new; coinciding with $H_{\rho}$ in reflexive Banach spaces and with $W$ in $l^{1}$, it seems to be not very interesting.

5. Description of $W$ BY a Class OF NONCOMPACt SETS

We saw in Proposition 4.2 that Wijsman convergence may be defined by types $p$ and $q$, taking $\mathfrak{X}$ such that $\mathscr{S}(E) \subset \mathfrak{X} \subset \mathscr{K}(E)$. A natural question is whether $W$ may be obtained by type $p$ and a class containing noncompact sets. The answer is positive when $E$ is a n.v.s. We shall see that we may take $\mathfrak{X}=\{\bar{B}(u, \rho) \mid u \in E, \rho>0\}$. The next example shows that this statement is not true in metric spaces.

Example 5.1. Let $E=l^{2}, A=\left\{\left(1+\frac{1}{p}\right) e_{p} \mid p \in \mathbf{N}^{*}\right\}, A_{n}=A \cup\left\{-e_{n}\right\}, F=$ $A \cup\left\{-e_{n} \mid n \in \mathbf{N}^{*}\right\} \cup\{0\}$ and $d$ the metric on $F$ inherited from $l^{2}$. Then, $W-\lim \left(A_{n}\right)=A$ in $l^{2}$, hence in $F$, but $\left(A_{n}\right)$ does not converge to $A$ with respect to $\mathfrak{X}(p), \mathfrak{X}=\{\bar{B}(u, \rho) \mid u \in F, \rho>0\}$.

Indeed, for $u \in E$, $d\left(u, A_{n}\right)=\min \left\{d(u, A),\left\|u+e_{n}\right\|\right\}=\min \left\{d(u, A),\left(\|u\|^{2}+2\left(u \mid e_{n}\right)+1\right)^{1 / 2}\right\} ;$ hence $\lim d\left(u, A_{n}\right)=\min \left\{d(u, A),\left(\|u\|^{2}+1\right)^{1 / 2}\right\}$. But

$$
\begin{aligned}
d(u, A) & \leq\left\|u-\left(1+\frac{1}{p}\right) e_{p}\right\| \\
& =\left(\|u\|^{2}-2\left(1+\frac{1}{p}\right)\left(u \mid e_{p}\right)+\left(1+\frac{1}{p}\right)^{2}\right)^{1 / 2} \quad \text { for } p \in \mathbf{N}^{*} .
\end{aligned}
$$


For $p \rightarrow \infty$ we get $d(u, A) \leq\left(\|u\|^{2}+1\right)^{1 / 2}$. Hence $A=W-\lim \left(A_{n}\right)$ in $l^{2}$ and therefore in $(F, d)$.

On the other hand $\bar{B}_{F}(0,1)=\{0\} \cup\left\{-e_{n} \mid n \in \mathbf{N}^{*}\right\}$ and $d\left(A_{n}, \bar{B}_{F}(0,1)\right)=0$ for every $n$.

$$
\begin{aligned}
d\left(A, \bar{B}_{F}(0,1)\right) & =\min \left\{\inf \left\{1+\frac{1}{p} \mid p \in \mathbf{N}^{*}\right\}, \inf \left\{\left\|e_{n}+\left(1+\frac{1}{p}\right) e_{p}\right\| \mid n, p \in \mathbf{N}^{*}\right\}\right\} \\
& =\min \left\{1, \inf \left\{\left(1+2\left(1+\frac{1}{p}\right)\left(e_{n} \mid e_{p}\right)+\left(1+\frac{1}{p}\right)^{2}\right)^{1 / 2} \mid n, p \in \mathbf{N}^{*}\right\}\right\} \\
& =\min \{1, \sqrt{2}\}=1 .
\end{aligned}
$$

Therefore $\left(A_{n}\right)$ does not $\mathfrak{X}(p)$-converge to $A$.

In the rest of this section $E$ is a n.v.s. We shall need the following elementary result (probably known, but without reference):

Lemma 5.2. Let $(E, d)$ be a metric space, $A, X$ be in $\mathscr{F}(E)$ and $\varepsilon>0$. Then

$$
d(A, \bar{B}(X, \varepsilon)) \leq d(A, X) \leq d(A, \bar{B}(X, \varepsilon))+\varepsilon .
$$

Moreover, if $E$ is a n.v.s. then

$$
d(A, \bar{B}(X, \varepsilon))=\max \{d(A, X)-\varepsilon, 0\} .
$$

Proof. As $X \subset \bar{B}(X, \varepsilon), d(A, \bar{B}(X, \varepsilon)) \leq d(A, X)$. Let now $a \in A, x^{\prime} \in$ $\bar{B}(X, \varepsilon)$. Then for every $n \in \mathbf{N}^{*}$ there exists $x_{n} \in X$ such that $d\left(x^{\prime}, x_{n}\right) \leq \varepsilon+$ $1 / n$. Letting first $n$ to tend to infinity, then taking the infimum for $a \in A, x^{\prime} \in$ $\bar{B}(X, \varepsilon)$ we get $d(A, \bar{B}(X, \varepsilon)) \geq d(A, X)-\varepsilon$. It follows that $d(A, \bar{B}(X, \varepsilon)) \geq$ $\max \{d(A, X)-\varepsilon, 0\}$.

Now let $E$ be a n.v.s. and $\delta>0$. If $d(A, X)<\varepsilon$ then $A \cap \bar{B}(X, \varepsilon) \neq \varnothing$ and so $d(A, \bar{B}(X, \varepsilon))=0=\max \{d(A, X)-\varepsilon, 0\}$. Let now $d(A, X) \geq \varepsilon$ and $\delta>0$; there exist $x \in X$ and $a \in A$ such that $d(A, X)>\|a-x\|-\delta$. As $d(A, X) \geq \varepsilon,\|a-x\| \geq \varepsilon$. There exists $\lambda \in[0,1]$ such that for $y=$ $(1-\lambda) a+\lambda x$ we have $\|y-x\|=\varepsilon$, i.e. $y \in \bar{B}(X, \varepsilon)$, and $\|a-y\|=\|a-x\|-\varepsilon$. Therefore $d(A, X)>\|a-x\|-\delta=\|a-y\|+\varepsilon-\delta$ for all $\delta>0$, and so $d(A, X) \geq d(A, \bar{B}(X, \varepsilon))+\varepsilon$.

Proposition 5.3. Let $(E, d)$ be a metric space, $\varnothing \neq \mathfrak{X} \subset \mathscr{F}(E)$ and $\mathfrak{X}_{0}=$ $\{\bar{B}(X, \varepsilon) \mid X \in \mathfrak{X}, \varepsilon>0\}$. Then $(\mathscr{F}, \mathfrak{X}, p) \subset\left(\mathscr{F}, \mathfrak{X}_{0}, p\right)$. Moreover, if $E$ is a n.v.s. then $(\mathscr{F}, \mathfrak{X}, p)=\left(\mathscr{F}, \mathfrak{X}_{0}, p\right)$.

Proof. Let first $(E, d)$ be a metric space. To show that $(\mathscr{F}, \mathfrak{X}, p) \subset\left(\mathscr{F}, \mathfrak{X}_{0}, p\right)$, it is sufficient to show that for $X \in \mathfrak{X}, r>0$ and $\delta \in] 0, r[$ we have

$$
\left\{(A, B) \mid p_{\bar{B}(X, \delta)}(A, B)<r-\delta\right\} \subset\left\{(A, B) \mid p_{X}(A, B)<r\right\} .
$$

Indeed, let $A, B \in \mathscr{F}(E)$ such that $p_{\bar{B}(X, \delta)}(A, B)<r-\delta$. Then, by Lemma 5.2, we have

$$
\begin{aligned}
d(A, X)-d(B, X) & \leq d(A, \bar{B}(X, \delta))+\delta-d(B, \bar{B}(X, \delta)) \\
& \leq p_{\bar{B}(X, \delta)}(A, B)+\delta<r .
\end{aligned}
$$

Changing $A$ and $B$ in the above relation we get $p_{X}(A, B)<r$.

Let now $E$ be a n.v.s., $X \in \mathfrak{X}$ and $\varepsilon>0$. Then, by using Lemma 5.2 and the elementary inequality $|\max \{\alpha, 0\}-\max \{\beta, 0\}| \leq|\alpha-\beta|$, we have

$$
\begin{aligned}
p_{\bar{B}(X, \varepsilon)}(A, B) & =|\max \{d(A, X)-\varepsilon, 0\}-\max \{d(B, X)-\varepsilon, 0\}| \\
& \leq p_{X}(A, B)
\end{aligned}
$$


for all $A, B \in \mathscr{F}(E)$. Therefore we have also $(\mathscr{F}, \mathfrak{X}, p) \supset\left(\mathscr{F}, \mathfrak{X}_{0}, p\right)$ in this case.

Corollary 5.4. Let $E$ be a n.v.s. and $\left(A_{i}\right)_{i \in I} \subset \mathscr{F}(E), A \in \mathscr{F}(E)$. Then $A=W-\lim \left(A_{i}\right)$ iff for all $u \in E, \rho>0$,

$$
d(A, \bar{B}(u, \rho))=\lim d\left(A_{i}, \bar{B}(u, \rho)\right) .
$$

In other words, $W$ is defined by $(\mathscr{F}, \mathfrak{X}, p)$ with $\mathfrak{X}=\{\bar{B}(u, \rho) \mid u \in E, \rho>0\}$.

Corollary 5.5. Let $E$ be a n.v.s., and $\mathfrak{X} \subset \mathscr{F}(E)$ be such that for $X \in \mathfrak{X}$ and $\varepsilon>0, \bar{B}(X, \varepsilon) \in \mathfrak{X}$. Then $(\mathscr{F}, \mathfrak{X}, p)=(\mathscr{F}, \mathscr{Y}, p)$, where $\mathscr{Y}=\{X \in \mathfrak{X} \mid$ $\operatorname{int}(X) \neq \varnothing\}$.

The families $\mathfrak{X}=\mathscr{F}(E), \mathscr{B}(E), \mathscr{C}(E), \mathscr{C}(E) \cap \mathscr{B}(E)$ have the property from Corollary 5.5. If $E$ is a reflexive Banach space, then $\mathscr{K}_{\sigma}(E)$ has the same property.

\section{TOPOLOGIES DEFINED BY TYPE $p$}

Let $(E, d)$ be a metric space and $\mathscr{A}, \mathfrak{X} \subset \mathscr{F}(E)$. The topology $\mathfrak{X}(p)$ generated by the uniformity $(\mathscr{A}, \mathfrak{X}, p)$ is the weakest for which the functions $A \rightarrow d(A, X)$ are continuous for all $X \in \mathfrak{X}$. Writing the condition for these functions to be lower semicontinuous and upper semicontinuous we get

Proposition 6.1. The topology $\mathfrak{X}(p)$ on $\mathscr{A}$ is generated by the following two families of set

$$
\begin{aligned}
\mathscr{D}(X, \varepsilon) & =\{A \in \mathscr{A} \mid d(A, X)<\varepsilon\} \\
& =\{A \in \mathscr{A} \mid A \cap B(X, \varepsilon) \neq \varnothing\}, \\
\mathscr{E}(X, \varepsilon) & =\{A \in \mathscr{A} \mid d(A, X)>\varepsilon\} \\
& =\{A \in \mathscr{A} \mid \exists \alpha>0: A \cap B(X, \varepsilon+\alpha)=\varnothing\},
\end{aligned}
$$

when $X \in \mathfrak{X}$ and $\varepsilon>0$.

When $E$ is a n.v.s., by using Lemma 5.2 , it is obvious that $\mathscr{E}(X, \varepsilon)=$ $\{A \in \mathscr{A} \mid d(A, B(X, \varepsilon))>0\}$.

Such generators are specific for "hit and miss" topologies as the classical Vietoris and Fell topologies (see [Mi, Fe, Ef, Mr1, 2, KT, Ma]). For the topologies corresponding to $M, L, Z$ (which will be defined later), we indicate the papers of G. Beer [Be1, 2, 3 and BLLN]; for $S$ see [SoZ1].

When $\mathscr{S}(E) \subset \mathfrak{X}$ the topology generated by $\{\mathscr{D}(X, \varepsilon) \mid X \in \mathfrak{X}, \varepsilon>0\}$ may be described only by the topology of $E$.

Proposition 6.2. If $\mathfrak{X} \supset \mathscr{S}(E)$ then the topology generated by $\{\mathscr{D}(X, \varepsilon) \mid X \in \mathfrak{X}$, $\varepsilon>0\}$ equals that generated by $\{\{A \in \mathscr{A} \mid A \cap U \neq \varnothing\} \mid U \subset E, U$ open $\}$.

Proof. As $B(X, \varepsilon)$ is open, it is sufficient to show that for $U \subset E, U$ open, $\{A \in \mathscr{A} \mid A \cap U \neq \varnothing\}=U^{-}$is in the topology $\mathscr{T}$ generated by $\{\mathscr{D}(X, \varepsilon) \mid X \in \mathfrak{X}$, $\varepsilon>0\}$. For $u \in U$ there exists $\varepsilon_{u}>0$ such that $B\left(u, \varepsilon_{u}\right) \subset U$. Thus $U=\bigcup_{u \in U} B\left(u, \varepsilon_{u}\right)$ and so $U^{-}=\{A \in \mathscr{A} \mid A \cap U \neq \varnothing\}=\{A \in \mathscr{A} \mid \exists u \in U$, $\left.A \cap B\left(u, \varepsilon_{u}\right) \neq \varnothing\right\}=\bigcup_{u \in U} \mathscr{D}\left(\{u\}, \varepsilon_{u}\right) \in \mathscr{T}$.

These very simple generators $\left(U^{-}, U\right.$ open) are found in the topologies of Vietoris, Fell, Mosco, $L, Z, \ldots$ But for $\mathfrak{X}=\mathscr{H}(E)$ (when $E$ is a n.v.s.), 
$\operatorname{dim}(E) \geq 2$ (see [SoZ1]) the adequate generators are $\{A \in \mathscr{A} \mid A \cap D(\varphi, \alpha) \neq$ $\varnothing\}$, where $D(\varphi, \alpha)=\{u \in E \mid \varphi(u)<\alpha\}, \varphi \in E^{\prime} \backslash\{0\}, \alpha \in \mathbf{R}$.

\section{CONVERGENCES OF TYPE $p$ FOR CLASSES$$
\mathfrak{X}=\mathscr{F}(E) \text { AND } \mathfrak{X}=\mathscr{F}_{\sigma}(E)
$$

Fisher convergence, $Z$. This convergence was introduced recently in [Fil, $\mathrm{DaK}]$ and studied in [Fi2, BaP, So2, BLLN, SP, Sh].

Let $(E, d)$ be a metric space.

Definition 7.1. The net $\left(A_{i}\right)_{i \in I} \subset \mathscr{F}(E)$ converges to $A \in \mathscr{F}(E)$ in the sense of Fisher, denoted $A=Z-\lim \left(A_{i}\right)$, if

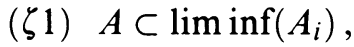

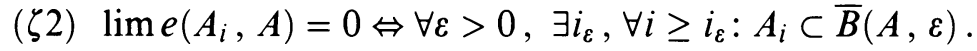

Note that between $H_{\rho}$ and $Z$ there are, generally, no implications. For instance, in $E=l^{2}, A_{n}=E_{n}, B_{n}=U \cap E_{n}: E=Z-\lim \left(A_{n}\right)$ and $U=$ $Z-\lim \left(B_{n}\right)$ but $\left(A_{n}\right)$ and $\left(B_{n}\right)$ are not $H_{\rho}$-convergent, while in $E=\mathbf{R}^{2}$, $A_{n}=\left\{\left(s, \frac{1}{n} s\right) \mid s \in \mathbf{R}\right\}, A=\{(s, 0) \mid s \in \mathbf{R}\}$, we have $A=H_{\rho}-\lim \left(A_{n}\right)$ but $\left(A_{n}\right)$ is not $Z$-convergent.

In the next result we show that $Z$ is exactly $\mathscr{F}(p)$; this gives an interesting theoretical status to $Z$ as the finest convergence of type $p$. On the other hand, $Z$ is a possible substitute for $H_{\rho}$, as the examples in $l^{2}$ above show.

Let us denote by $U(E, \mathbf{R})$ the class of uniformly continuous functions $f$ : $E \rightarrow \mathbf{R}$.

Theorem 7.2. Let the net $\left(A_{i}\right)_{i \in I} \subset \mathscr{F}(E)$ and $A \in \mathscr{F}(E)$. The following statements are equivalent:

(1) $Z-\lim \left(A_{i}\right)=A$,

(2) $\lim \mu\left(f, A_{i}\right)=\mu(f, A)$ for all $f \in U(E, \mathbf{R})$ (where $\mu(f, B)=$ $\inf \{f(x) \mid x \in B\}$,

(3) $\mathscr{F}(p)-\lim \left(A_{i}\right)=A$,

(4) $\lim e\left(A_{i}, X\right)=e(A, X)$ for all $X \in \mathscr{F}(E)$.

Proof. (1) $\Rightarrow(2)$ Suppose that $A=Z-\lim \left(A_{i}\right)$ and take $f \in U(E, \mathbf{R})$. From ( $\zeta 1)$, by using Theorem 3.3, we have $\lim \sup \mu\left(f, A_{i}\right) \leq \mu(f, A)$. Suppose that $\liminf \mu\left(f, A_{i}\right)<l<\mu(f, A)$. Then $J=\left\{i \in I \mid \mu\left(\bar{f}, A_{i}\right)<l\right\}$ is cofinal. For each $i \in J$ there exists $u_{i} \in A_{i}$ such that $f\left(u_{i}\right)<l$.

Consider $0<\varepsilon<\mu(f, A)-l$. As $f$ is uniformly continuous, there exists $\delta>0$ such that $|f(u)-f(v)|<\varepsilon$ for $u, v \in E, d(u, v)<\delta$. By $(\zeta 2)$, there exists $i_{0}$ such that for $i \geq i_{0}$ we have $A_{i} \subset \bar{B}(A, \delta / 2) \subset B(A, \delta)$. As $J$ is cofinal, there exists $i \in J, i \geq i_{0}$. For this $i$ we get $a_{i} \in A$ such that $d\left(u_{i}, a_{i}\right)<\delta$ and therefore $\mu(f, A) \leq f\left(a_{i}\right)<f\left(u_{i}\right)+\varepsilon<l+\varepsilon<\mu(f, A)$, a contradiction. Therefore, $\mu(f, A) \leq \liminf \mu\left(f, A_{i}\right)$.

$(2) \Rightarrow(3)$. Let $X \in \mathscr{F}(E)$. As $d(\cdot, X) \in U(E, \mathbf{R})$ we have that $\lim d\left(A_{i}, X\right)$ $=d(A, X)$, i.e. $\mathscr{F}(p)-\lim \left(A_{i}\right)=A$.

(3) $\Rightarrow(1)$. Once again by Theorem 3.3 , we have that $(\zeta 1)$ holds. Suppose that $(\zeta 2)$ is not true. Then there exists $\varepsilon_{0}>0$ such that $J=\left\{i \in I \mid A_{i} \not \subset\right.$ $\left.\bar{B}\left(A, \varepsilon_{0}\right)\right\}$ is cofinal. Thus, for every $i \in J$, there exists $a_{i} \in A_{i}$ such that $d\left(a_{i}, A\right)>\varepsilon_{0}$. Consider $X=\operatorname{cl}\left\{a_{i} \mid i \in J\right\} \in \mathscr{F}(E)$. Then $\lim d\left(A_{i}, X\right)=$ 
$d(A, X)$. As for $i \in J, d\left(A_{i}, X\right)=0$, it follows that $d(A, X)=0$, a contradiction, because $d(A, X)=\inf \left\{d\left(a_{i}, A\right) \mid i \in J\right\} \geq \varepsilon_{0}>0$.

$(2) \Rightarrow(4)$. For $X \in \mathscr{F}(E),-d(\cdot, x) \in U(E, \mathbf{R})$ and therefore $\lim e\left(A_{i}, X\right)$ $=-\lim \left(\inf \left\{-d(x, X) \mid x \in A_{i}\right\}\right)=-\inf \{-d(x, X) \mid x \in A\}=e(A, X)$.

(4) $\Rightarrow$ (1). Taking $X=A$, we get that $\lim e\left(A_{i}, A\right)=0$, i.e. ( $\left.\zeta 2\right)$ holds. Let now $a \in A$. We may suppose that $E \neq\{a\}$ and let $0<\varepsilon<\sup \{d(x, y) \mid y \in$ $E\}(>0)$. Consider $X=\{x \in E \mid d(x, a) \geq \varepsilon\} \neq \varnothing$. Then for $B \in \mathscr{F}(E)$ we have $e(B, X)=\sup _{x \in B} d(x, X)=\sup _{x \in B \cap B(a, \varepsilon)} d(x, X)$. As $\lim e\left(A_{i}, X\right)=$ $\lim \left(\sup _{x \in A_{i} \cap B(a, \varepsilon)} d(x, X)\right)=e(A, X) \geq \varepsilon$, there exists $i_{\varepsilon}$ such that $A_{i} \cap$ $B(a, \varepsilon) \neq \varnothing$ for $i \geq i_{\varepsilon}$, i.e. $d\left(a, A_{i}\right)<\varepsilon$ for $i \geq i_{\varepsilon}$. This shows that $a \in$ $\lim \inf \left(A_{i}\right)$.

Remark. The implication $(1) \Rightarrow(3)$ of Theorem 7.2 is proven in $[\mathrm{BaP}]$; the equivalence (1) $\Leftrightarrow(3)$ appears in [BLLN] in a topological framework, as well as $(3) \Leftrightarrow(4)$ in [BLu]. The result concerning optimization is new as well as the proof of the theorem.

Let $u \in E$ and $Y \in \mathscr{B}(E)$. We take $\rho(u, Y)=\sup \{d(u, v) \mid v \in Y\}$ and for $X \in \mathscr{F}(E), \rho(X, Y)=\inf \{\rho(u, Y) \mid u \in X\}$ (i.e. the Chebyshev radius of $Y$ with respect to $X)$; also for $X \in \mathscr{B}(E)$ we consider $\tau(X, Y)=$ $\sup \{\rho(u, Y) \mid u \in X\}$ (the common diameter of $X$ and $Y$ ).

Corollary 7.3. Let $\left(A_{i}\right)_{i \in I} \subset \mathscr{F}(E)$ be a net and $A \in \mathscr{F}(E)$. Suppose that $A=$ $Z-\lim \left(A_{i}\right)$. Then $\lim \rho\left(A_{i}, X\right)=\rho(A, X)$ for every $X \in \mathscr{B}(E)$. Moreover, if $\left(A_{i}\right)_{i \in I} \subset \mathscr{B}(E)$ and $A \in \mathscr{B}(E)$ then $\lim \tau\left(A_{i}, X\right)=\tau(A, X)$ for all $X \in$ $\mathscr{B}(E)$.

Proof. It is sufficient to observe that for $Y$ bounded we have $\mid \rho(u, Y)-$ $\rho(v, Y) \mid \leq d(u, v)$ for all $u, v \in E$, and apply Theorem 7.2 for $\rho(\cdot, X)$ and $-\rho(\cdot, X)$.

Convergence $\mathscr{F}_{\sigma}(p)$. When $E$ is a n.v.s. it is natural to consider also the class $\mathfrak{X}=\mathscr{F}_{\sigma}(E)$. We shall see later (in this section) that $Z=\mathscr{F}(p)$ and $\mathscr{F}_{\sigma}(p)$ are different convergences.

The next result establishes a relation between $\mathscr{F}_{\sigma}(p)$ and Mosco convergence, $M$. Let us remind that the sequence $\left(A_{n}\right)_{n \in \mathbf{N}}$ of nonempty subsets of the n.v.s. $E$ Mosco converges to $A \in 2^{E} \backslash\{\varnothing\}$, denoted $A=M-\lim \left(A_{n}\right)$ if:

$$
\sigma\left(E, E^{\prime}\right)-\lim \sup \left(A_{n}\right) \subset A \subset \liminf \left(A_{n}\right),
$$

where $\sigma\left(E, E^{\prime}\right)-\lim \sup \left(A_{n}\right)=\left\{x \in E \mid\right.$ there exists $\left(x_{k}\right)_{k \in K} \subset\left(A_{n}\right)_{n \in \mathbf{N}}, K$ infinite, $\left.x=\sigma\left(E, E^{\prime}\right)-\lim \left(x_{k}\right)\right\}$.

Proposition 7.4. Let $E$ be a reflexive Banach space and $\left(A_{n}\right)_{n \in \mathrm{N}} \subset \mathscr{F}_{R}(E)$, $A \in \mathscr{F}(E)$ for some $R>0$. If $A=M-\lim \left(A_{n}\right)$ then $A=\mathscr{F}_{\sigma}(p)-\lim \left(A_{n}\right)$.

Proof. Let $X \in \mathscr{F}_{\sigma}$. As $A \subset \liminf \left(A_{n}\right)$, by using Theorem 3.3, we have that $\lim \sup d\left(A_{n}, X\right) \leq d(A, X)$. Suppose that $\liminf d\left(A_{n}, X\right)<l<d(A, X)$. Then $K=\left\{n \in \mathbf{N} \mid d\left(A_{n}, X\right)<l\right\}$ is infinite. For every $n \in K$ there exist $a_{n} \in A_{n}$ and $x_{n} \in X$ such that $\left\|a_{n}-x_{n}\right\|<l$. From the hypothesis we have that $\left(a_{n}\right) \subset \bar{B}(0, R)$. As $X$ is reflexive, taking a subsequence if necessary, there exists $a \in E$ such that $a=\sigma\left(E, E^{\prime}\right)-\lim _{n \in K}\left(a_{n}\right)$. As $\left(a_{n}\right)$ is bounded, also $\left(x_{n}\right)_{n \in K}$ is bounded and $\sigma\left(E, E^{\prime}\right)-\lim _{n \in K}\left(x_{n}\right)=x \in E$. It follows that 
$a \in A$ and $x \in X$. On the other hand, from the weak lower semicontinuity of the norm, we have $\|a-x\| \leq l$, i.e. $d(A, X) \leq l$, a contradiction. Therefore $\lim d\left(A_{n}, X\right)=d(A, X)$.

We shall see in $\S 9$ that the converse implication is valid even without boundedness of $\left(A_{n}\right)$, but with $A \in \mathscr{F}_{\sigma}(E)$, for general n.v.s.

The next example shows that in general the convergences $\mathscr{F}(p)$ and $\mathscr{F}_{\sigma}(p)$ are distinct.

Example 7.5. Let $E=l^{2}, A_{n}=\left[0, e_{n}\right], A=\{0\}$. Then $A=M-\lim \left(A_{n}\right)$ and so $A=\mathscr{F}_{\sigma}(p)-\lim \left(A_{n}\right)$, but $\left(A_{n}\right)$ is not $\mathscr{F}(p)$-convergent.

Indeed, it is simple to see that $A=M-\lim \left(A_{n}\right)$, and so, by Proposition 7.4, $A=\mathscr{F}_{\sigma}(p)-\lim \left(A_{n}\right)$. But for $X=S(0,1) \in \mathscr{F}, d\left(A_{n}, X\right)=0$ while $d(A, X)=1$.

In Proposition 3.4, we showed that every increasing net is convergent for $\mathfrak{X}(p)$, but for a decreasing net $\left(A_{i}\right)$ we showed only that, in the case $\mathscr{S} \subset \mathfrak{X}$, if it is convergent its limit is $\bigcap_{i \in I} A_{i}$.

The next result establishes a sufficient condition for the convergence of a decreasing net.

Proposition 7.6. Let $E$ be a reflexive Banach space and $\left(A_{i}\right)_{i \in I} \subset \mathscr{F}_{\sigma}(E)$ be a decreasing net. If there exists $i_{0}$ such that $A_{i_{0}}$ is bounded then $A=\bigcap_{i \in I} A_{i}$ is nonempty and $A=\mathscr{F}_{\sigma}(p)-\lim \left(A_{i}\right)$.

Proof. Because $\left(A_{i}\right)_{i \in I}$ is decreasing, $A=\bigcap_{i \geq i_{0}} A_{i}$. As $A_{i_{0}}$ is $\sigma\left(E, E^{\prime}\right)$ compact (being bounded and $\sigma\left(E, E^{\prime}\right)$ closed), it follows that $A \neq \varnothing$. From $A \subset A_{i}$, it follows that

$$
d(A, X) \geq d\left(A_{i}, X\right)
$$

and therefore $d(A, X) \geq \limsup d\left(A_{i}, X\right)$ for every $\varnothing \neq X \subset E$. Suppose that for some $X \in \mathscr{F}_{\sigma}(E)$ we have $\liminf d\left(A_{i}, X\right)<l<d(A, X)$. Let $J=\left\{i \in I \mid i \geq i_{0}, d\left(A_{i}, X\right)<l\right\} ; J$ is cofinal. For every $i \in J$ there exist $a_{i} \in A$ and $x_{i} \in X$ such that $\left\|x_{i}-a_{i}\right\|<l$. As $\left(a_{i}\right)_{i \in J} \subset A_{i_{0}},\left(a_{i}\right)_{i \in J}$ has a $\sigma\left(E, E^{\prime}\right)$-convergent subnet, i.e. there exist a directed set $K$ and $\varphi: K \rightarrow J$ such that for every $i \in J$ there exists $k_{i} \in K$ with $\varphi(k) \geq i$ for $k \geq k_{i}$ and $a=\sigma\left(E, E^{\prime}\right)-\lim \left(a_{\varphi(k)}\right)$. Because $\left(a_{i}\right)_{i \in I}$ is bounded, it follows that $\left(x_{i}\right)_{i \in J}$ is bounded, too. We may suppose that there exists $x=\sigma\left(E, E^{\prime}\right)-\lim \left(x_{\varphi(k)}\right)$. Therefore $x \in X$. Let us note that $a \in A$.

Indeed, let $i \in I$. As $J$ is cofinal, there exists $j \in J$ such that $j \geq i$. From the definition of $\varphi$, there exists $k_{j}$ such that $\varphi(k) \geq j \geq i$ for all $k \geq k_{j}$. Therefore $a_{\varphi(k)} \in A_{i}$ for all $k \geq k_{j}$ and so $a=\lim \left(a_{\varphi(k)}\right) \in A_{i}$. Hence $a \in A$. By the lower semicontinuity of the norm we get $d(A, X) \leq\|a-x\| \leq l$, a contradiction.

A similar result one can obtain for $\left(A_{i}\right)_{i \in I} \subset \mathscr{F}(E)$ a decreasing net, where $(E, d)$ is a metric space, for $\mathscr{F}(p)$, if we suppose that the closed balls of $E$ are compact.

The convergence $\mathscr{F}_{\sigma}(p)$, which is strictly weaker than $Z$, is new. Its restriction to $\mathscr{A}=\mathscr{F}_{\sigma}(E)$ may be interesting for the convergence of nets of nonconvex subsets of $E$. 


\section{CONVERGENCES OF TYPE $p$ FOR CLASSES OF CLOSED AFFINE SUBSETS}

Throughout this section $E$ is a n.v.s. Before introducing the convergences announced in the title of the section we remind two convergences.

Scalar convergence, $S$.

Definition 8.1. The net $\left(A_{i}\right)_{i \in I} \subset \mathscr{C}(E)$ scalar converges to $A \in \mathscr{C}(E)$, denoted $A=S-\lim \left(A_{i}\right)$, if

for all $\varphi \in E^{\prime}: s_{A}(\varphi)=\lim s_{A_{i}}(\varphi)$ (in $\overline{\mathbf{R}}$ ), or equivalently:

for all $\varphi \in S^{\prime}(0,1): s_{A}(\varphi)=\lim s_{A_{i}}(\varphi)$, where $s_{A}(\varphi)=\sup \varphi(A)$, i.e. $s_{A}$ is the support function of $A$.

We mention the references concerning $S$ : [Wi, SaW, DBM, He, So2, Be3, SoZ1, FiL].

We restricted to $\mathscr{C}(E)$ because $s_{X}=s_{\overline{\operatorname{conv}}(X)}$.

Linear convergence, $L$. This notion was introduced by G. Beer in [Be3] and, independently, by C. Hess in his thesis [He].

Definition 8.2. The net $\left(A_{i}\right)_{i \in I} \subset \mathscr{C}(E) \quad L$-converges to $A \in \mathscr{C}(E)$, denoted $A=L-\lim \left(A_{i}\right)$, if

$$
A=W-\lim \left(A_{i}\right) \text { and } A=S-\lim \left(A_{i}\right) .
$$

Next we introduce on $\mathscr{A}=\mathscr{C}(E)$ the uniformities corresponding to type $p$ and class $\mathfrak{X}, \mathfrak{X}$ being $\mathscr{C}(E)$ and classes of closed affine subsets as $\mathscr{V}(E), \mathscr{V}_{f}(E)$, $\mathscr{V}_{c}(E), \mathscr{S}(E), \mathscr{H}(E)$. We may also consider classes of linear subspaces $\mathscr{V}^{0}$, $\mathscr{V}_{f}^{0}, \mathscr{V}_{c}^{0}, \mathscr{H}^{0}$. We have the following inclusions:

$$
\begin{gathered}
(\mathscr{C}, \mathscr{H}, p) \subset\left(\mathscr{C}, \mathscr{V}_{c}, p\right) \subset(\mathscr{C}, \mathscr{V}, p) \subset(\mathscr{C}, \mathscr{C}, p) \subset\left(\mathscr{C}, \mathscr{F}_{\sigma}, p\right) \\
(\mathscr{C}, S, p) \subset\left(\mathscr{C}, \mathscr{V}_{f}, p\right) \subset(\mathscr{C}, \mathscr{V}, p) \subset(\mathscr{C}, \mathscr{C}, p) .
\end{gathered}
$$

The next result was proven in [SoZ1]. We give here another proof.

Theorem 8.3. Let $\left(A_{i}\right)_{i \in I} \subset \mathscr{C}(E)$ be a net and $A \in \mathscr{C}(E)$. Then

$$
A=S-\lim \left(A_{i}\right) \Leftrightarrow A=\mathscr{H}(p)-\lim \left(A_{i}\right) .
$$

Proof. Every $H \in \mathscr{H}$ can be written as $H=\{x \in E \mid \varphi(x)=\alpha\}$ with $\varphi \in$ $S^{\prime}(0,1)$ and $\alpha \in \mathbf{R}$. As for $X \in \mathscr{C}(E), \varphi(X) \subset \mathbf{R}$ is an interval, we easily get that

$$
d(X, H)=\inf \{d(x, H) \mid x \in X\}=\inf \{|\varphi(x)-\alpha| \mid x \in X\}=d(\alpha, \overline{\varphi(X)}) .
$$

Thus

$$
\begin{aligned}
A=\mathscr{H}(p)-\lim \left(A_{i}\right) & \Leftrightarrow \forall H \in \mathscr{H}: \lim d\left(A_{i}, H\right)=d(A, H) \\
& \Leftrightarrow \forall \varphi \in S^{\prime}, \forall \alpha \in \mathbf{R}: \lim d\left(\alpha, \overline{\varphi\left(A_{i}\right)}\right)=d(\alpha, \overline{\varphi(A)}) \\
& \left.\Leftrightarrow \forall \varphi \in S^{\prime}: \overline{\varphi(A)}\right)=K-\lim \left(\overline{\varphi\left(A_{i}\right)}\right) \quad(\text { see }[\mathrm{SaW}]) \\
& \Leftrightarrow S-\lim \left(A_{i}\right)=A \quad(\text { see }[\operatorname{SoZ} \text {, Corollary 2]). } \quad
\end{aligned}
$$

Remark. The convergence $S$ is also defined by another uniformity (see [SoZ1]) nonequivalent to $(\mathscr{C}, \mathscr{H}, p)$, defined by 


$$
\tilde{p}_{\varphi}(A, B)=\left|\left(f \circ s_{A}\right)(\varphi)-\left(f \circ s_{B}\right)(\varphi)\right|, \quad \varphi \in S^{\prime},
$$

where $f(t)=\frac{t}{1+|t|}, t \in \mathbf{R}= \pm 1$ for $t= \pm \infty$.

We may also consider the uniformity $(\mathscr{F}, \mathscr{H}, p)$ to extend $S$ to $\mathscr{F}(E)$, but this uniformity is not separated.

In [Be3, Theorem 3.7], G. Beer showed that the topology corresponding to $L$ is the weakest on $\mathscr{C}(E)$ for which the function $\mathscr{C} \times \mathscr{C} \ni(A, B) \rightarrow d(A, B) \in \mathbf{R}$ is continuous. We show here that $L$ is $\mathscr{C}(p)$ on $\mathscr{C}(E)$, which is weaker than the above mentioned result.

Theorem 8.4 (G. Beer). Let $\left(A_{i}\right)_{i \in I} \subset \mathscr{C}(E)$ be a net and $A \in \mathscr{C}(E)$. Then

$$
A=L-\lim \left(A_{i}\right) \text { iff } A=\mathscr{C}(p)-\lim \left(A_{i}\right) \text {. }
$$

Proof. As $\mathscr{S} \subset \mathscr{C}$ and $\mathscr{H} \subset \mathscr{C}$ the implication " $\Leftarrow$ " is obvious. Let us show the converse implication. As $A=W-\lim \left(A_{i}\right)$ we have that $A \subset \lim \inf \left(A_{i}\right)$, and therefore, by Theorem 3.3, $d(A, X) \geq \limsup d\left(A_{i}, X\right)$ for $X \in \mathscr{C}$. Let $X \in \mathscr{C}$ such that $0<\delta=d(A, X)=d(0, X-A)$; therefore $B(0, \delta) \cap(X-A)=$ $\varnothing$. Using a separation theorem (see [Ho]), there exists $\varphi \in S^{\prime}$ such that

$$
\delta=\sup _{u \in B(0, \delta)} \varphi(u) \leq \inf \{\varphi(x-a) \mid x \in X, a \in A\}=\inf _{x \in X} \varphi(x)-s_{A}(\varphi) .
$$

Let $l<\delta$. Then $s_{A}(\varphi) \leq \inf _{x \in X} \varphi(x)-\delta<\inf _{x \in X} \varphi(x)-l$.

As $\lim s_{A_{i}}(\varphi)=s_{A}(\varphi)$, we get $i_{0}$ such that for $i \geq i_{0}$ we have $s_{A_{i}}(\varphi)<$ $\inf _{x \in X} \varphi(x)-l \Leftrightarrow l<\inf \left\{\varphi(x-a) \mid x \in X, a \in A_{i}\right\}$ for all $i \geq i_{0}$ hence $l \leq\|x-a\|$ for all $x \in X, a \in A_{i}, i \geq i_{0}$, hence $l \leq d\left(A_{i}, X\right)$ for $i \geq i_{0}$.

Therefore, $d(A, X) \leq \liminf d\left(A_{i}, X\right)$. As the case $d(A, X)=0$ is obvious, we have that $A=\overline{\mathscr{C}}(p)-\lim \left(A_{i}\right)$.

Corollary 8.5. On $\mathscr{C}(E)$ the convergences $L,(\mathscr{S} \cup \mathscr{H})(p), \mathscr{V}(p), \mathscr{C}(p)$ coincide.

It is remarkable that the conjunction of $\mathscr{S}(p)$, corresponding to closed affine subsets (c.a.s.) of dimension 0 , and $\mathscr{H}(p)$, corresponding to c.a.s. of codimension 1 , implies the convergences $\mathscr{V}(p)$ and $\mathscr{C}(p)$. Simple examples show that convergence corresponding to c.a.s. of dimension $n$ (codimension $n-1$ ) does not imply the convergence corresponding to c.a.s. of dimension $n+1$ (codimension $n$ ).

It is interesting to study the convergence $S_{\rho}$ defined similarly as $H_{\rho}$ : the net $\left(A_{i}\right) \subset \mathscr{C}(E) S_{\rho}$-converges to $A \in \mathscr{C}(E)$ if there exists $\rho_{0}>0$ such that for all $\rho \geq \rho_{0}, \lim s_{A_{i} \cap \rho U}(\varphi)=s_{A \cap \rho U}(\varphi)$ for all $\varphi \in E^{\prime}$ (see Remark following Definition 4.4).

Concerning the convergences $\mathscr{V}_{f}(p)$ and $\mathscr{V}_{c}(p)$ we give three examples:

Example 8.6. Let $E=l^{2}, A_{n}=\operatorname{span}\left\{e_{n}\right\}, A=\{0\} .\left(A_{n}\right)$ does not $S$-converge but some computations show that $\mathscr{V}_{f}(p)-\lim \left(A_{n}\right)=A$.

Example 8.7. Let $E=l^{2}, A_{n}=\left\{e_{n}\right\}, A=\{0\} .\left(A_{n}\right)$ does not $W$-converge but $A=\mathscr{V}_{c}(p)-\lim \left(A_{n}\right)$.

Example 8.8. Let $E=l^{2}, A_{n}=\left[e_{1}, e_{n}\right], A=\left[0, e_{1}\right], B=\left\{e_{1}\right\}$. Then $S-\lim \left(A_{n}\right)=A, K-\lim \left(A_{n}\right)=B$ and $W-\lim \left(A_{n}\right)$ does not exist.

The convergences $\mathscr{V}_{f}(p)$ and $\mathscr{V}_{c}(p)$ seem to be new for $\operatorname{dim}(E)=\infty$, while for $\operatorname{dim}(E)<\infty$ we have $\mathscr{V}(p)=\mathscr{V}_{f}(p)=\mathscr{V}_{c}(p)=L$. 


\section{CONVERGENCES OF TYPE $p$ FOR CLASSES OF BOUNDED SETS}

In this section we are interested by the uniformity $(\mathscr{F}, \mathscr{B}, p)$ when $E$ is a metric space and by uniformities $(\mathscr{C}, \mathscr{B}, p),(\mathscr{C}, \mathscr{B} \cap \mathscr{F}, p),(\mathscr{C}, \mathscr{C} \cap$ $\mathscr{B}, p),\left(\mathscr{C}, \mathscr{K}_{\sigma}, p\right),\left(\mathscr{C}, \mathscr{K}_{\sigma} \cap \mathscr{C}, p\right)$ when $E$ is a n.v.s.

First let us consider a metric space $(E, d)$. In this case we consider the uniformity $(\mathscr{F}, \mathscr{B}, p)$. We shall show that the corresponding convergence $\mathscr{B}(p)$ is just the convergence introduced by $\mathrm{P}$. Shunmugaraj [Sh] in his thesis and then in [ShP] (for n.v.s.). The corresponding topology appears in the paper of H. Attouch, D. Azé, and G. Beer [AAB]. See also [BLu].

Definition 9.1. Let $(E, d)$ be a metric space. The net $\left(A_{i}\right)_{i \in I} \subset \mathscr{F}(E)$ $Z_{\rho}$-converges to $A \in \mathscr{F}(E)$, denoted $A=Z_{\rho}-\lim \left(A_{i}\right)$, if

$$
A \subset \liminf \left(A_{i}\right),
$$

$$
\forall X \in \mathscr{B}(E), \forall \varepsilon>0, \exists i_{\varepsilon} \in I, \forall i \geq i_{\varepsilon}: A_{i} \cap X \subset \bar{B}(A, \varepsilon) .
$$

In the initial definition in [Sh, ShP], considered in n.v.s. for sequences, the condition $\left(\zeta_{\rho} 2\right)$ was given in the form

$$
\exists \rho_{0}>0, \forall \rho \geq \rho_{0}, \forall \varepsilon>0, \exists n_{0}, \forall n \geq n_{0}: A_{n} \cap \rho U \subset \bar{B}(A, \varepsilon),
$$

which is equivalent to $\left(\zeta_{\rho} 2\right)$ in this context.

Let us note that condition $\left(\zeta_{\rho} 2\right)$ is equivalent to

$\left(\zeta_{\rho} 2\right)^{\text {bis }} \quad$ for each bounded $\operatorname{net}\left(a_{i}\right)_{i \in I} \subset\left(A_{i}\right)_{i \in I}, \lim d\left(a_{i}, A\right)=0$.

Also note that $Z_{\rho}$ is for $Z$ as $H_{\rho}$ for $H$, which explains the notation (in [SoZ2] we denoted by SP this convergence).

Let us denote by $V(E, \mathbf{R})$ the class of uniformly continuous functions $f$ : $E \rightarrow \mathbf{R}$ which are inf-bounded, i.e. $\{x \in E \mid f(x) \leq \alpha\}$ is bounded for every $\alpha \in \mathbf{R}$, and by $W(E, \mathbf{R})$ the class of functions $f: E \rightarrow \mathbf{R}$ which are uniformly continuous on bounded subsets and inf-bounded.

Theorem 9.2. Let $\left(A_{i}\right)_{i \in I} \subset \mathscr{F}(E)$ be a net and $A \in \mathscr{F}(E)$. Then the following statements are equivalent:

(1) $Z_{\rho}-\lim \left(A_{i}\right)=A$,

(2) $\lim \mu\left(f, A_{i}\right)=\mu(f, A)$ for every $f \in W(E, \mathbf{R})$,

(3) $\lim \mu\left(f, A_{i}\right)=\mu(f, A)$ for every $f \in V(E, \mathbf{R})$,

(4) $\mathscr{B}(p)-\lim \left(A_{i}\right)=A$.

Proof. (1) $\Rightarrow(2)$. Let $f \in W(E, \mathbf{R})$. From $\left(\zeta_{\rho} 1\right)$, by using Theorem 3.3, we have $\lim \sup \mu\left(f, A_{i}\right) \leq \mu(f, A)$. Suppose that $\liminf \mu\left(f, A_{i}\right)<l<$ $\mu(f, A)$. Then $J=\left\{i \in \bar{I} \mid \mu\left(f, A_{i}\right)<l\right\}$ is cofinal. For every $i \in J$ there exists $a_{i} \in A_{i}$ such that $f\left(a_{i}\right)<l$. Let $X=\{u \in E \mid f(u) \leq l\} ; X \in \mathscr{B}(E)$. Consider $0<\varepsilon \leq \mu(f, A)-l$. As $F$ is uniformly continuous on $\bar{B}(X, 1)$ :

$$
\exists \eta \in(0,1), \forall u, v \in \bar{B}(X, 1): d(u, v)<\eta \Rightarrow|f(u)-f(v)|<\varepsilon / 2 .
$$

From $\left(\zeta_{\rho} 2\right)$ there exists $i_{0}$ such that for $i \geq i_{0}$ we have $X \cap A_{i} \subset \bar{B}(A, \eta / 2)$ $\subset B(A, \eta)$, and so there exists $a \in A$ such that $d\left(a, a_{i}\right)<\eta<1$. Moreover $a \in \bar{B}(X, 1)$. Hence 


$$
\mu(f, A) \leq f(a)<f\left(a_{i}\right)+\varepsilon<l+\varepsilon \leq \mu(f, A),
$$

a contradiction.

The implication $(2) \Rightarrow(3)$ is obvious.

$(3) \Rightarrow(4)$. Let $X \in \mathscr{B}(E)$ and take $f=d(\cdot, X)$. Then $f \in V(E, \mathbf{R})$. Hence $\lim d\left(A_{i}, X\right)=d(A, X)$.

(4) $\Rightarrow(1)$. Taking $X=\{u\}$ we get that $A=W-\lim \left(A_{i}\right)$ and therefore $\left(\zeta_{\rho} 1\right)$ holds. Suppose that $\left(\zeta_{\rho} 2\right)$ is false. Then there exist $X \in \mathscr{B}(E)$ and $\varepsilon_{0}>0$ such that $J=\left\{i \in I \mid X \cap A_{i} \not \subset \bar{B}\left(A, \varepsilon_{0}\right)\right\}$ is cofinal. For every $i \in I$ let $x_{i} \in A_{i} \cap X$ such that $d\left(x_{i}, A\right) \geq \varepsilon_{0}$ and consider $Y=\operatorname{cl}\left\{x_{i} \mid i \in J\right\} \in$ $\mathscr{B}(E)$. It follows that $\varepsilon_{0} \leq d(A, Y)=\lim d\left(A_{i}, Y\right)=\lim \inf d\left(A_{i}, Y\right)=0$, a contradiction.

Remark. The equivalence $(1) \Rightarrow(4)$ is established independently in [BLu].

Corollary 9.3. Let the net $\left(A_{i}\right)_{i \in I} \subset \mathscr{F}(E) Z_{\rho}$-converges to $A \in \mathscr{F}(E)$. Then $\lim \rho\left(A_{i}, X\right)=\rho(A, X)$ for every $X \in \mathscr{B}(X)$.

Proof. Apply Theorem 9.2 to $f=\rho(\cdot, X) \in W(E, \mathbf{R})$.

Corollary 9.4. The convergence $Z_{\rho}$ is weaker than $Z$ and $H_{\rho}$ but finer than $W$.

This result is known (see [Sh, ShP]).

The convergence $Z_{\rho}$ seems to be, in our opinion, very interesting in the case of metric spaces, being much finer than $W$ and much weaker than $H_{\rho}$. In the case of n.v.s. there are also other substitutes for $H_{\rho}$ as we shall see in the sequel. The results of [Sh and ShP] show its utility in optimization. Let us consider now the case of a n.v.s. $E$ and denote by $Z(E, \mathbf{R})$ the class of continuous functions $f: E \rightarrow \mathbf{R}$ which are $\sigma\left(E, E^{\prime}\right)$-inf-compact, i.e. $\{x \in E \mid f(x) \leq \alpha\}$ is $\sigma\left(E, E^{\prime}\right)$-compact for all $\alpha \in \mathbf{R}$.

Proposition 9.5. Let $\left(A_{i}\right)_{i \in I} \subset \mathscr{F}(E)$ be a net and $A \in \mathscr{F}(E)$.

(1) If $\mathscr{K}_{\sigma}-\lim \left(A_{i}\right)=A \in \mathscr{F}_{\sigma}$ then $\lim \mu\left(f, A_{i}\right)=\mu(f, A)$ for all $f \in$ $Z(E, \mathbf{R})$.

(2) If $E$ is a reflexive Banach space and $\lim \mu\left(f, A_{i}\right)=\mu(f, A)$ for every $f \in Z(E, \mathbf{R})$ then $A=\mathscr{K}_{\sigma}-\lim \left(A_{i}\right)$.

Proof. (1) Let $f \in Z(E, \mathbf{R})$; as $A=\mathscr{K}_{\sigma}-\lim \left(A_{i}\right), A=W-\lim \left(A_{i}\right)$ and so $\lim \sup \mu\left(f, A_{i}\right) \leq \mu(f, A)$. Suppose that $\liminf \mu\left(f, A_{i}\right)<l<\mu(f, A)$. Then $J=\left\{i \in I \mid \mu\left(f, A_{i}\right)<l\right\}$ is cofinal. The set $X=\{x \in E \mid f(x) \leq l\} \in \mathscr{K}_{\sigma}$. As $d\left(A_{i}, X\right)=0$ for $i \in J$, it follows that $0=d(A, X)=d(0, X-A)$. But in our hypothesis $X-A \in \mathscr{F}_{\sigma} \subset \mathscr{F}$ so that $0 \in X-A$ i.e. $A \cap X \neq \varnothing$, a contradiction. Therefore, $\mu(f, A)=\lim \mu\left(f, A_{i}\right)$.

(2) Let $X \in \mathscr{K}_{\sigma}$. Then $d(\cdot, X) \in Z(E, \mathbf{R})$. Thus we get $A=\mathscr{K}_{\sigma}-$ $\lim A_{i}$.

Another result of the same type is

Proposition 9.6. Let $\left(A_{n}\right)_{n \in \mathbf{N}} \subset \mathscr{F}(E)$ and $A \in \mathscr{F}(E)$. If $A=M-\lim \left(A_{n}\right)$ then $\mu(f, A)=\lim \mu\left(f, A_{n}\right)$ for every $f \in Z(E, \mathbf{R})$.

Proof. As above we get $\mu(f, A) \geq \lim \sup \mu\left(f, A_{n}\right)$. With the notations in the proof of Proposition 9.5, $J$ is infinite and $X \in \mathscr{K}_{\sigma}$. Let $a_{n} \in A_{n}$ be such that 
$f\left(a_{n}\right)<l$ for $n \in J$. Taking a subsequence if necessary, we may suppose that there exists $a=\sigma\left(E, E^{\prime}\right)-\lim _{n \in J}\left(a_{n}\right)$. Then $a \in A \cap X$, a contradiction.

Corollary 9.7. Let $E$ be a reflexive Banach space, $\left(A_{n}\right)_{n \in \mathbf{N}} \subset \mathscr{F}(E)$ and $A \in$ $\mathscr{F}(E)$. If $A=M-\lim \left(A_{n}\right)$ then $A=\mathscr{K}_{\sigma}-\lim \left(A_{n}\right)$.

Proof. Take in Proposition $9.6 f=d(\cdot, X) \in Z(E, \mathbf{R})$ for $X \in \mathscr{K}_{\sigma}$.

Remarks. (1) One can find Proposition 9.6, with a different proof, in [Sh], while Corollary 9.7 can be found in [So2, Proposition 70; Be2, Theorem 3.3]. The implication $M \Rightarrow W$ when $E$ is reflexive is proved in [Sol].

(2) Corollary 9.7 is false for $\mathscr{K}_{\sigma}$ replaced by $\mathscr{B}$ (see Example 7.5 ) or by $\mathscr{F}_{\sigma}$. Indeed, take $E=l^{2}, A_{n}=\left[0, n e_{n}\right], A=\{0\} ; A=M-\lim \left(A_{n}\right)$ [Sol, So2], but for $X=\left\{u \in E \mid \sum_{k \geq 1} \frac{1}{k} u_{k}=1\right\} \in \mathscr{F}_{\sigma}$ we have $d\left(A_{n}, X\right)=0$ and $d(A, X)=\sqrt{6} / \pi \neq 0$.

(3) Corollary 9.7 is also false for $E$ nonreflexive because $M$ does not generally imply $W$ (see [BoFi, Theorem 2.2(b)], and examples in [BaP, So2]).

The next result states sufficient conditions for Mosco-convergence of a sequence.

Proposition 9.8. Let $\left(A_{n}\right)_{n \in \mathbf{N}} \subset \mathscr{F}(E)$ and $A \in \mathscr{F}(E)$. Consider the following hypothesis:

(a) $K_{\sigma}(p)-\lim \left(A_{n}\right)=A$ and $A \in \mathscr{F}_{\sigma}$,

(b) $(\mathscr{C} \cap \mathscr{B})(p)-\lim \left(A_{n}\right)=A$ and $A$ is convex,

(c) $\left(K_{\sigma} \cap \mathscr{C}\right)(p)-\lim \left(A_{n}\right)=A, A$ is convex and $E$ is a Banach space.

If $(\mathrm{a})$ or (b) or (c) holds then $A=M-\lim \left(A_{n}\right)$. In particular $\mathscr{B}(p)$, i.e. $Z_{\rho}$, and $\left(\mathscr{B} \cap \mathscr{F}_{\sigma}\right)(p)$ imply $M$.

Proof. In each of the three cases we have $A=W-\lim \left(A_{n}\right)$ and so $A \subset$ $\lim \inf \left(A_{n}\right)$. So we must only show that $A \supset \sigma\left(E, E^{\prime}\right)-\lim \sup \left(A_{n}\right)$. So let $a=\sigma\left(E, E^{\prime}\right)-\lim _{n \in P}\left(a_{n}\right)$, where $P \subset \mathbf{N}$ is infinite and $a_{n} \in A_{n}$ for $n \in P$. Consider first the case (a). If $Q=\left\{n \in P \mid a_{n} \in A\right\}$ is infinite then $a \in A$. In the contrary case we may suppose that $Q=\varnothing$. Let $X=\left\{a, a_{n} \mid n \in P\right\}$; $X \in \mathscr{K}_{\sigma}$. As $d\left(A_{n}, X\right)=0$ for $n \in P$, it follows that $d(A, X)=0$. As in the proof of Proposition 9.5, we get $A \cap X \neq \varnothing$, whence $a \in A$.

Suppose now that (b) holds and $a \notin A$. By a separation theorem, there exists $\varphi \in S^{\prime}(0,1)$ such that $\varphi(a)>l>\sup \{\varphi(x) \mid x \in A\}$. As $\lim _{n \in P} \varphi\left(a_{n}\right)=\varphi(a)$ we may suppose that $\varphi\left(a_{n}\right) \geq l$ for every $n \in P$. Taking now $Y=\overline{\operatorname{conv}}(X)$, $X$ from (a), $Y \in \mathscr{C} \cap \mathscr{B}$. As above we get $d(A, Y)=0$. As $Y \subset\{x \mid \varphi(x) \geq l\}$ and $A\{x \mid \varphi(x) \leq \sup \varphi(A)\}$ we get also that $d(A, Y) \geq l-\sup \varphi(A)>0$, a contradiction.

In the case (c), the set $Y$ is even $\sigma\left(E, E^{\prime}\right)$-compact by the Krein Theorem (see [DS, p. 434], for instance). The rest of the proof is as in case (b).

Remark. (a) $\Rightarrow M$ and (c) $\Rightarrow M$ are already known in reflexive setting with $A_{n}, A$ convex sets [Be2, BP].

The above results give the following:

Corollary 9.9 (G. Beer and D. Pai). Let $E$ be a reflexive Banach space and $A, A_{n} \in \mathscr{C}(E), n \in \mathbf{N}$. Then: $A=M-\lim \left(A_{n}\right)$ iff $A=\mathfrak{X}(p)-\lim \left(A_{n}\right)$ where $\mathfrak{X}=\mathscr{K}_{\sigma}$ or $\mathfrak{X}=\mathscr{K}_{\sigma} \cap \mathscr{C}$. 
The convergence $\mathscr{B}_{\sigma}(p)=\left(\mathscr{B} \cap \mathscr{F}_{\sigma}\right)(p)$, being between $Z_{\rho}$ and $M$, could be interesting. $\mathscr{B}_{\sigma}(p)$ is for $\mathscr{F}_{\sigma}$ as $\mathscr{B}(p)$, i.e. $Z_{\rho}$, for $\mathscr{F}(p)$, i.e. $Z$.

Remark. It is not difficult to prove (cf. Theorem 9.2) that for $A, A_{i} \in \mathscr{F}$ we have

$$
(\mathscr{C} \cap \mathscr{B})(p)-\lim \left(A_{i}\right)=A \text { iff } \lim \mu\left(f, A_{i}\right)=\mu(f, A)
$$

for all $f: E \rightarrow \mathbf{R}$ convex, inf-bounded, and uniformly continuous on bounded subsets.

A similar result holds for $L$ and $f$ convex and uniformly continuous.

The next examples point out the differences between some convergences.

Let $E$ be a nonreflexive Banach space. In [BoFi] is constructed a sequence $\left(A_{n}\right) \subset \mathscr{C} \cap \mathscr{K}$, uniformly bounded, and $A \in \mathscr{C} \cap \mathscr{K}$ such that $A=M-$ $\lim \left(A_{n}\right)$, but $\left(A_{n}\right)$ is not $W$-convergent (and not $S$-convergent, [SoZ1]). That example shows that even on $\mathscr{A}=(\mathscr{C} \cap \mathscr{K})(E)$ the convergence $M$ is different from the convergences studied here as $\mathscr{B}_{\sigma}(p),(\mathscr{C} \cap \mathscr{B})(p), \mathscr{K}_{\sigma}(p),\left(\mathscr{K}_{\sigma} \cap\right.$ $\mathscr{C})(p)$.

Earlier we had the sequence $\left(A_{n}\right)$ with $A_{n}=\left[0, e_{n}\right]$ and $A=\{0\}$ in $l^{2}$. In this case $A=\mathfrak{X}(p)-\lim \left(A_{n}\right)$ for $\mathfrak{X}=\mathscr{S}, \mathscr{K}_{\sigma} \cap \mathscr{C}, \mathscr{K}_{\sigma}, \mathscr{B}_{\sigma}, \mathscr{C} \cap \mathscr{B}$, convergences that are equivalent to $M$ on $l^{2}$ (which has Kadec property, see [BoFi]), and also for $\mathfrak{X}=\mathscr{F}_{\sigma}$, but $\left(A_{n}\right)$ is not $Z_{\rho}=\mathscr{B}(p)$-convergent and therefore it is not $Z$ or $H_{\rho}$-convergent.

Even in a reflexive Banach space $W$ does not imply $M$ (see [Be4 and BoFi]). Hence $W$ does not imply $(\mathscr{C} \cap \mathscr{B})(p), \mathscr{K}_{\sigma}(p),\left(\mathscr{K}_{\sigma} \cap \mathscr{C}\right)(p)$ which are finer than $M$.

Let $E=l^{1}, A_{n}=\left[0, e_{n}\right], A=\{0\}$ as in Example 4.5. Consider $X=$ $\overline{\operatorname{conv}}\left\{e_{1}, \ldots, e_{n}, \ldots\right\} \in \mathscr{C} \cap \mathscr{B}$. Then $d\left(A_{n}, X\right)=0$ but $d(A, X)=1$. Thus $\left(A_{n}\right)$ is not $(\mathscr{C} \cap \mathscr{B})(p)$-convergent. But $\left(A_{n}\right) W$-converges to $A$ (Proposition $4.2)$, for $\mathscr{K}(p)=\mathscr{K}_{\sigma}(p)$, and therefore converges for $\left(\mathscr{K}_{\sigma} \cap \mathscr{C}\right)(p)$. In fact in $l^{1}$ the convergences $\mathscr{S}, \mathscr{K}_{\sigma} \cap \mathscr{C}$, and $\mathscr{K}_{\sigma}$ are equivalent. On the other hand in $l^{1} \quad M$ and $K$ are equivalent for sequences and therefore $\sup (W, M)$ does not imply $(\mathscr{C} \cap \mathscr{B})(p)$.

We have no example to show that $\mathscr{K}_{\sigma}(p)$ and $\left(\mathscr{C} \cap \mathscr{K}_{\sigma}\right)(p)$ are not equivalent.

\section{RecApitulation}

First let $(E, d)$ be a metric space and $\mathscr{A}=\mathscr{F}(E)$. In this case, we dispose of the following convergences $H=\mathscr{F}(q)=\{E\}(q)$ that is finer than $H_{\rho}=$ $\mathscr{B}(q)$, which is finer than $Z_{\rho}=\mathscr{B}(p)$, which is finer, as its turn, than $W=$ $\mathscr{S}(p)=\mathscr{K}(p)=\mathscr{K}(q)$; at last $Z=\mathscr{F}(p)$ is finer than $Z_{\rho}$

The relations between them are obtained trivially by comparing their classes or using the inequality $p \leq q$.

Let now $E$ be a n.v.s. On $\mathscr{A}=\mathscr{C}(E)$ one has the implications mentioned in the next figure. Except those concerning $M$ all the implications are trivial and obtained by comparing their classes or the inequality $p \leq q$. We remember that $S=\mathscr{H}(p)$ and $L=\sup (W, S)=\mathscr{C}(p)=\mathscr{V}(p)$. The convergences $\mathscr{V}_{f}(p)$ and $\mathscr{V}_{c}(p)$ do not appear in this figure. The implications concerning $M$ refer to sequences, while " $\leftarrow--$ " signifies that $E$ must be complete.

When $E$ is a reflexive Banach space and its dual has Kadec property, we have $W \Rightarrow M$ on $\mathscr{C}(E)$ and so $W \Leftrightarrow M$ (see [BoFi]). 


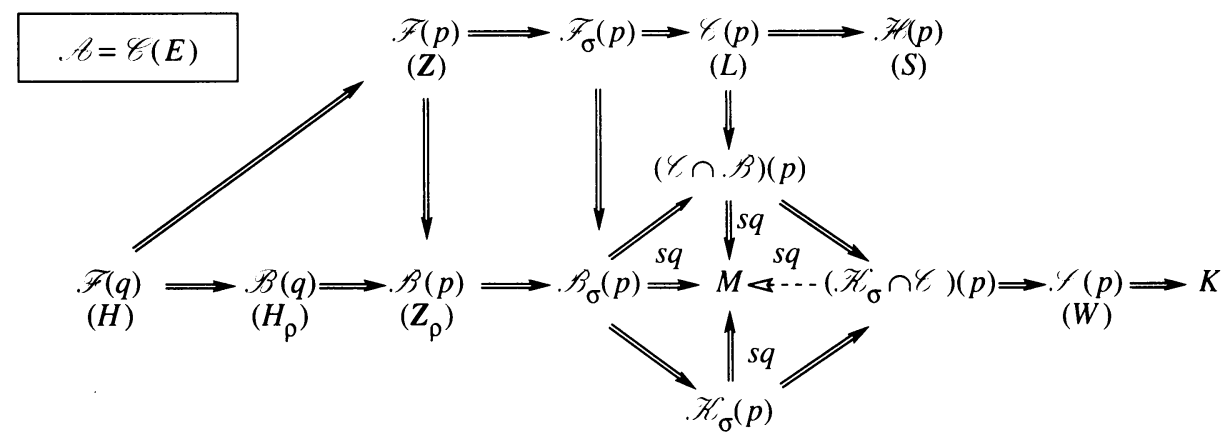

When $\operatorname{dim}(E)<\infty$, on $\mathscr{C}(E)$, we have $H_{\rho} \Leftrightarrow Z_{\rho} \Leftrightarrow W \Leftrightarrow K$ and so only five different convergences remain $H, Z, W, S, L$.

When $E=\mathbf{R}$ all the convergences, excepting $H\left(A_{n}=[-n, n]\right)$, coincide. The former assertion is proved by the following examples in $E=\mathbf{R}^{2}$.

Example 10.1.

$$
A_{n}=\left\{\left(s, \frac{s}{n}\right) \mid s \in \mathbf{R}\right\}, \quad A=\{(s, 0) \mid s \in \mathbf{R}\}, \quad A=H_{\rho}-\lim \left(A_{n}\right),
$$

but does not converge for $S, H$ (and so for $Z, L$ ).

\section{Example 10.2.}

$$
A_{n}=\{(s, t) \mid s \leq 0, t \geq n(s+1)\}, \quad A=\{(s, t) \mid s \leq 0\}, \quad B=\{(s, t) \mid s \leq-1\}
$$

(see [Wi, SaW]). We have $A=S-\lim \left(A_{n}\right)$ and $B=W-\lim \left(A_{n}\right)$. So $S$ does not imply $L$ or $Z$.

\section{COMPLETENESS FOR TYPES $p$ AND $q$}

The problem of the completeness of $(\mathscr{A}, \mathfrak{X}, q)$ is solved, using general results from [Bou3], where the problem of metrisability of the induced topology is also solved. For $H_{\rho}$ see [ALW, Lu1, Be5].

The semimetric spaces $(\mathscr{A}, \mathfrak{X}, p)$ are not, generally, complete, as we shall see in the sequel. We consider only the case $\mathscr{S} \subset \mathfrak{X}$ when the limit is unique if it exists, and only the sequential completeness.

Note that the sequence $\left(A_{n}\right) \subset \mathscr{A}$ is Cauchy iff $\left(d\left(A_{n}, X\right)\right)$ is convergent in $\mathbf{R}$ for every $X \in \mathfrak{X}$. Thus $(\mathscr{A}, \mathfrak{X}, p)$ is sequentially complete iff for every sequence $\left(A_{n}\right) \subset \mathscr{A}$ such that $\left(d\left(A_{n}, X\right)\right)$ is convergent in $\mathbf{R}$ for every $X \in \mathfrak{X}$ there exists $A \in \mathscr{A}$ such that $\lim d\left(A_{n}, X\right)=d(A, X)$ for every $X \in \mathfrak{X}$.

The next example shows that $(\mathscr{A}, \mathfrak{X}, p)$ is not complete for $\mathscr{S} \subset \mathscr{A}$ and $\mathscr{S} \subset \mathfrak{X} \subset \mathscr{H}$.

Example 11.1. $E=l^{2}, A_{n}=\left\{e_{n}\right\}$. For every $u \in E, d\left(u, A_{n}\right)=\left\|u-e_{n}\right\|=$ $\left(\|u\|^{2}-2\left(u \mid e_{n}\right)+1\right)^{1 / 2} \rightarrow\left(\|u\|^{2}+1\right)^{1 / 2}$, but $\left(A_{n}\right)$ is not $\mathscr{S}(p)$-convergent.

Note that $W$ can be described by a complete uniformity (in fact Polish) when $(E, d)$ is a Polish space (see [LeL, Be6]).

An interesting problem concerning the convergences $\mathfrak{X}(p)$ is to find complete uniformities, whose convergence be $\mathfrak{X}(p)$, when $(E, d)$ is complete. The answer is known for $W$ (loc. cit.) and for $S$ (see [DBM, SoZ1]). 
In order to show that $(\mathscr{A}, \mathfrak{X}, p)$ is not complete it is sufficient to construct an increasing sequence $\left(A_{n}\right) \subset \mathscr{A}$ such that $\operatorname{cl}\left(\bigcup_{n \in \mathbf{N}} A_{n}\right)$ is not in $\mathscr{A}$ or a decreasing sequence $\left(A_{n}\right) \subset \mathscr{A}$ such that $\bigcap_{n \in \mathbf{N}} A_{n} \neq \varnothing$ and $\left(A_{n}\right)$ does not converge to $A$. The following examples use these criteria.

Example 11.2. Let $E$ be a n.v.s., $A_{n}=\{0\} \cup\{x \mid\|x\| \geq n\}$. $\left(A_{n}\right)$ is decreasing with $\bigcap_{n \in \mathbf{N}} A_{n}=\{0\}=A$, but taking $X_{0}=\{\lambda y \mid \lambda \geq 1\}, y \neq 0, d\left(A_{n}, X_{0}\right)=0$, $d(A, X)=\|y\| \neq 0$. Thus $(\mathscr{A}, \mathfrak{X}, p)$ is not complete for $\mathscr{A}=\mathscr{F}(E)$ and $\mathfrak{X} \ni X_{0}$.

Example 11.3. Let $E=\mathbf{R}, A_{n}=[-n, n] .\left(A_{n}\right)$ is increasing and $\bigcup_{n \in \mathbf{N}} A_{n}=$ R. Thus $(\mathscr{A}, \mathfrak{X}, p)$ is not complete for $\mathscr{A} \supset \mathscr{K} \cap \mathscr{C}$ with $\mathbf{R} \notin \mathscr{A}$ and $\mathscr{S} \subset \mathfrak{X}$.

Example 11.4. Let $E=l^{2}, A_{n}=\overline{\operatorname{conv}}\left\{e_{n}, e_{n+1}, \ldots\right\} .\left(A_{n}\right)$ is decreasing and $\bigcap_{n \in \mathbf{N}} A_{n}=\{0\}=A$, but $d\left(A_{n}, S\right)=0$ while $d(A, S)=1$. Thus $(\mathscr{A}, \mathfrak{X}, p)$ is not complete for $\mathscr{A} \supset \mathscr{K}_{\sigma} \cap \mathscr{C}, S=S(0,1) \in \mathfrak{X}(\supset \mathscr{S})$.

Example 11.5. Let $E=l^{2}, A_{n}=E_{n}^{\perp} .\left(A_{n}\right)$ is decreasing and $\bigcap_{n \in \mathbf{N}} A_{n}=$ $\{0\}=A$. For $X \subset l^{2}$,

$$
d(A, X)=\inf _{x \in X}\|x\|, \quad d\left(A_{n}, X\right)=\inf _{x \in X}\left\|x-\left(x-P_{n}(x)\right)\right\|=\inf _{x \in X}\left\|P_{n}(x)\right\| .
$$

Then for $X=\left\{n e_{n} \mid n \geq 1\right\} \in \mathscr{F}_{\sigma}$ we have $d(A, X)=1$, while $d\left(A_{n}, X\right) \leq$ $\left\|P_{n}\left((n+1) e_{n+1}\right)\right\|=0$. Thus $(\mathscr{A}, \mathfrak{X}, p)$ is not complete for $\mathscr{A} \supset \mathscr{V}_{c}$ and $\mathfrak{X} \supset \mathscr{F}_{\sigma}$.

If $E$ is a finite-dimensional n.v.s. there are only five distinct convergences: $H, Z, W, S, L$, the last two referring to convex sets. There are known results concerning the completeness of $H, W$ (loc. cit.) and $S, L$ [DBM, SoZ1]. The next result refers to the convergence $Z$.

Theorem 11.1. Let $E$ be a finite-dimensional n.v.s. and $\left(A_{n}\right) \subset \mathscr{B}(E)$ be such that $\left(d\left(A_{n}, X\right)\right)$ is convergent for every $X \in \mathscr{F}(E)$. Then there exists $A \in$ $\mathscr{F}(E)$ such that $A=Z-\lim \left(A_{n}\right)$.

Proof. Taking $X=\{0\}$ we get that $\left(d\left(0, A_{n}\right)\right)$ is convergent and therefore bounded. For every $n$ take $a_{n} \in A_{n}$ such that $\left\|a_{n}\right\|=d\left(0, A_{n}\right)$. As $\left(a_{n}\right)$ is bounded, it has a convergent subsequence. Therefore $A=\lim \sup \left(A_{n}\right) \neq \varnothing$. Let $a \in A$, then $\left(d\left(a, A_{n}\right)\right)$ is convergent and $\liminf d\left(a, A_{n}\right)=0$, whence $\lim d\left(a, A_{n}\right)=0$ and so $A \subset \liminf \left(A_{n}\right)$. Hence $A=K-\lim \left(A_{n}\right)$. Suppose that $\left(A_{n}\right)$ does not $Z$-converge to $A$, i.e. ( $\left.\zeta 2\right)$ does not hold. Then there exists $\varepsilon>0$ and $P \subset \mathbf{N}$ infinite such that $A_{n} \backslash B(A, 2 \varepsilon) \neq \varnothing$ for $n \in P$. Let us take $D_{n}=A_{n} \backslash B(A, \varepsilon)$ for $n \in P$. Our aim is to construct $X \in \mathscr{F}$ such that $\left(d\left(A_{n}, X\right)\right)$ is not convergent. Let $\alpha_{n}=\inf \left\{\|u\| \mid u \in D_{n}\right\}$ and $\beta_{n}=$ $\sup \left\{\|u\| \mid u \in D_{n}\right\}$. As $A_{n}$ is bounded, $\beta_{n}<\infty$. We have that $\lim \left(\alpha_{n}\right)=$ $\infty$; otherwise $\left(\alpha_{n}\right)$ contains a bounded subsequence, and consequently there exists $\left(u_{n}\right)_{n \in Q} \subset\left(D_{n}\right) \quad\left(Q \subset P, Q\right.$ infinite) such that $\left(u_{n}\right)_{n \in Q}$ is bounded. As $\operatorname{dim}(E)<\infty$ we may suppose that there exists $u=\lim _{n \in Q}\left(u_{n}\right)$. It follows that $u \in A$, contradicting the fact that $d\left(u_{n}, A\right) \geq \varepsilon$ for every $n \in Q$. Taking a subsequence if necessary, we may suppose that for every $n \in \mathbf{N}$ we have

$$
A_{n} \backslash B(A, 2 \varepsilon) \neq \varnothing, \quad D_{n}=A_{n} \backslash B(A, \varepsilon) \quad \text { and } \quad \alpha_{n+1} \geq \beta_{n}+1 .
$$

It follows that $\beta_{n-1}<\beta_{n-1}+1 \leq \alpha_{n} \leq \beta_{n} \leq \alpha_{n+1}-1<\alpha_{n+1}$ i.e. $\left(\alpha_{n}\right)$ and $\left(\beta_{n}\right)$ are (strictly) increasing sequences. For every $n \in \mathbf{N}$ there exists 
$u_{n} \in A_{n} \backslash B(A, 2 \varepsilon)$. Let us take $X=\operatorname{cl}\left\{u_{2}, u_{4}, \ldots, u_{2 n}, \ldots\right\} \in \mathscr{F}$. Thus $d\left(A_{2 k}, X\right)=0$ for every $k \in \mathbf{N}$ and $d\left(A_{2 k+1}, X\right)=\inf \left\{d\left(u_{2 n}, A_{2 k+1}\right) \mid n \geq 1\right\}$.

But $A_{2 k+1}=\left(A_{2 k+1} \cap B(A, \varepsilon)\right) \cup D_{2 k+1}$, whence

$$
\begin{aligned}
d\left(u_{2 n}, A_{2 k+1}\right) & \geq \min \left\{d\left(u_{2 n}, B(A, \varepsilon)\right), d\left(u_{2 n}, D_{2 k+1}\right)\right\} \\
& \geq \min \left\{\varepsilon, d\left(u_{2 n}, D_{2 k+1}\right)\right\} \geq \min \{\varepsilon, 1\} .
\end{aligned}
$$

Indeed, $\alpha_{2 n} \leq\left\|u_{2 n}\right\| \leq \beta_{2 n}$ and for $u \in D_{2 k+1}, \alpha_{2 k+1} \leq\|u\| \leq \beta_{2 k+1}$, whence for $2 n>2 k+1,\left\|u_{2 n}-u\right\| \geq\left\|u_{2 n}\right\|-\|u\| \geq \alpha_{2 n}-\beta_{2 k+1} \geq 1$, while for $2 n<2 k+1,\left\|u_{2 n}-u\right\| \geq\|u\|-\left\|u_{2 n}\right\| \geq \alpha_{2 k+1}-\beta_{2 n} \geq 1$.

Therefore $\left(d\left(A_{n}, X\right)\right)$ is not convergent, a contradiction. Hence $Z-\lim \left(A_{n}\right)$ $=A$.

Note that the above result does not state that $(\mathscr{B}, \mathscr{F}, p)$ is sequentially complete (see Example 11.3).

\section{SEMICONTINUITY AND CONTINUITY OF SOME OPERATIONS WITH SUBSETS}

For $\mathscr{A} \subset \mathscr{F}(E)$ and $\mathfrak{X} \subset \mathscr{F}(E)$ one can be interested in continuity properties of operations like $(A, B) \mapsto A \cup B,(A, B) \mapsto A \cap B, A \mapsto A \cap C$, $A \mapsto \Phi(A)$ (where $\Phi: E \rightarrow F$ is continuous), and for $E$ a n.v.s., $A \mapsto \overline{\operatorname{conv}}(A)$, $(A, B) \mapsto A+B,(\lambda, A) \mapsto \lambda A$.

In Theorem 3.3 we obtained that if $A \subset \liminf \left(A_{i}\right)$ then $\lim \sup d\left(A_{i}, X\right) \leq$ $d(A, X)$ for all $X \in \mathscr{F}(E)$. Let us call this property $Z^{+}$upper semicontinuity (u.s.c.). We have the following simple implications:

(1) If $\Phi: E \rightarrow F$ is continuous, with $E, F$ metric spaces and $A_{i}$, $A \in \mathscr{F}(E), A \subset \liminf \left(A_{i}\right)$ then $\overline{\Phi(A)} \subset \liminf \overline{\left(\Phi\left(A_{i}\right)\right)}$.

(2) If $E$ is a n.v.s. and $A_{i}, A, B_{i}, B \in \mathscr{F}(E)$ such that $A \subset \liminf \left(A_{i}\right)$, $B \subset \liminf \left(B_{i}\right)$ then $\operatorname{cl}(A+B) \subset \liminf \operatorname{cl}\left(A_{i}+B_{i}\right)$.

(3) If $E$ is a n.v.s. and $A_{i}, A \in \mathscr{F}(E), \lambda_{i}, \lambda \in \mathbf{R}$ such that $A \subset \liminf \left(A_{i}\right)$ and $\lim \left(\lambda_{i}\right)=\lambda$ then $\lambda A \subset \liminf \left(\lambda_{i} A_{i}\right)$.

(4) If $E$ is a n.v.s. and $A_{i}, A \in \mathscr{F}(E)$ such that $A \subset \liminf \left(A_{i}\right)$ then $\overline{\operatorname{conv}}(A) \subset \lim \inf \left(\overline{\operatorname{conv}}\left(A_{i}\right)\right)$.

(5) If $E$ is a n.v.s. and $C, A_{i}, A \in \mathscr{F}(E)$ are such that $A \subset \liminf \left(A_{i}\right)$ and $A \cap \operatorname{int}(C) \neq \varnothing$ then $A \cap \operatorname{int}(C) \subset \liminf \left(A_{i} \cap C\right)$. Moreover, if $A$ and $C$ are convex we have $A \cap C \subset \liminf \left(A_{i} \cap C\right)$.

In proving the above statements one must take into account that $\lim \inf \left(A_{i}\right)$ is always closed and it is convex when $A_{i}$ are convex. Moreover for (5) one can use that for $A$ and $C$ closed convex sets such that $A \cap \operatorname{int}(C) \neq \varnothing$ we have $A \cap C=\operatorname{cl}(A \cap \operatorname{int}(C))$.

We give two proper continuity results.

Proposition 12.1. Let $E$ be a metric space. Then the map $(A, B) \mapsto A \cup B$ from $(\mathscr{F}(E), \mathfrak{X}, p) \times(\mathscr{F}(E), \mathfrak{X}, p)$ to $(\mathscr{F}(E), \mathfrak{X}, p)$ is continuous.

Proof. Let $\left(A_{i}\right),\left(B_{i}\right) \subset \mathscr{F}(E)$ be $\mathfrak{X}(p)$-convergent to $A, B \in \mathscr{F}(E)$, respectively, and $X \in \mathfrak{X}$. Then

$$
\begin{aligned}
\lim d\left(A_{i} \cup B_{i}, X\right) & =\lim \left(\min \left\{d\left(A_{i}, X\right), d\left(B_{i}, X\right)\right\}\right) \\
& =\min \left\{\lim d\left(A_{i}, X\right), \lim d\left(B_{i}, X\right)\right\} \\
& =\min \{d(A, X), d(B, X)\}=d(A \cup B, X) .
\end{aligned}
$$


Proposition 12.2. Let $E$ be a n.v.s. and the family $\mathfrak{X}$ such that $\mathscr{S}(E) \subset \mathfrak{X} \subset$ $\mathscr{B}(E)$ and $\lambda X \in \mathfrak{X}$ for every $\lambda>0$, and $X \in \mathfrak{X}$. Then the map $(\lambda, A) \mapsto \lambda A$ from $(0, \infty) \times(\mathscr{F}(E), \mathfrak{X}, p)$ to $(\mathscr{F}(E), \mathfrak{X}, p)$ is continuous.

Proof. Let $\left(A_{i}\right) \subset \mathscr{F}(E) \mathfrak{X}(p)$-converges to $A \in \mathscr{F}(E)$ and $\left(\lambda_{i}\right) \subset(0, \infty)$, $\lim \left(\lambda_{i}\right)=\lambda \in(0, \infty)$. As $\mathscr{S} \subset \mathfrak{X}$ we have that $A \subset \liminf \left(A_{i}\right)$ and so $\lambda A \subset \liminf \left(\lambda_{i} A_{i}\right)$, which implies that $\lim \sup d\left(\lambda_{i} A_{i}, X\right) \leq d(\lambda A, X)$ for every $X \in \mathfrak{X}$. Let now $X \in \mathfrak{X}$ be fixed, $x \in X$ and $a_{i} \in A_{i}$. Then

$$
\begin{gathered}
\lambda_{i} d\left(A_{i}, X\right)-\left|\lambda-\lambda_{i}\right| \sup _{x \in X}\|x\| \leq \lambda_{i}\left\|a_{i}-x\right\|-\left|\lambda-\lambda_{i}\right|\|x\| \\
\leq\left\|\lambda_{i} a_{i}-\lambda_{i} x-\lambda x+\lambda_{i} x\right\|=\left\|\lambda_{i} a_{i}-\lambda x\right\|,
\end{gathered}
$$

whence

$$
\lambda_{i} d\left(A_{i}, X\right)-\left|\lambda-\lambda_{i}\right| \sup _{x \in X}\|x\| \leq d\left(\lambda_{i} A_{i}, \lambda X\right) .
$$

Therefore $\lambda d(A, X) \leq \liminf d\left(\lambda_{i} A_{i}, \lambda X\right)$. As $\lambda d(A, X)=d(\lambda A, \lambda X)$ and $\lambda X \in \mathfrak{X}$ we obtain that $d(\lambda A, X) \leq \liminf d\left(\lambda_{i} A_{i}, X\right)$ for every $X \in \mathfrak{X}$. Therefore $\mathfrak{X}(p)-\lim \left(\lambda_{i} A_{i}\right)=\lambda A$.

See [Be3] for a result concerning $L$.

The above result shows that the map $(\lambda, A) \mapsto \lambda A$ is $Z_{\rho}$-continuous. This statement is not true for $Z$.

Example 12.1. Let $E=\mathbf{R}^{2}, A=\left\{(s, t) \mid t \geq s^{2}\right\}$ and $\lambda_{n}=1+\frac{1}{n}:\left(\lambda_{n} A\right)$ does not $Z$-converge to $1 A=A$.

Indeed, for $\lambda>1, e(\lambda A, A)=\infty$.

\section{CONCLUdING REMARKS}

Throughout the paper we considered only two types, $p$ and $q$, and various classes $\mathfrak{X}$. We showed that a rich family of set convergences can be obtained in this way, the most part of known convergences being among them.

To the natural quantities in the metric space $(E, d): d(x, Y), d(X, Y)$, $e(X, Y), h(X, Y), \rho(x, Y), \rho(X, Y), \tau(X, Y)$ and

$$
k(X, Y)=\max \{\rho(X, Y), \rho(Y, X)\} \quad(x \in E, X, Y \in \mathscr{F}(E)),
$$

besides $p_{X}(A, B)$ and $q_{X}(A, B)$ we can also consider for $(A, B) \in \mathscr{A} \times \mathscr{A}$, $X \in \mathfrak{X}$,

$$
\begin{array}{rlrl}
q_{X}^{\prime}(A, B) & =\sup _{x \in X}|\rho(x, A)-\rho(x, B)| & & (\mathscr{A} \subset \mathscr{B}, \mathfrak{X} \subset \mathscr{F}), \\
r_{X}(A, B) & =|\rho(A, X)-\rho(B, X)| & & (\mathscr{A} \subset \mathscr{F}, \mathfrak{X} \subset \mathscr{B}), \\
r_{X}^{\prime}(A, B) & =|\rho(X, A)-\rho(X, B)| & & (\mathscr{A} \subset \mathscr{B}, \mathfrak{X} \subset \mathscr{F}), \\
s_{X}(A, B) & =|e(A, X)-e(B, X)| & & (\mathscr{A} \subset \mathscr{B}, \mathfrak{X} \subset \mathscr{F}), \\
s_{X}^{\prime}(A, B)=|e(X, A)-e(X, B)| & & (\mathscr{A} \subset \mathscr{F}, \mathfrak{X} \subset \mathscr{B}), \\
t_{X}(A, B) & =|\tau(A, X)-\tau(B, X)| & & (\mathscr{A} \subset \mathscr{B}, \mathfrak{X} \subset \mathscr{B}) .
\end{array}
$$

Using the well-known inequalities $|d(x, X)-d(y, X)| \leq d(x, y) \quad(X \in \mathscr{F})$, $|\rho(x, X)-\rho(y, X)| \leq d(x, y) \quad(X \in \mathscr{B})$ which are direct consequences of the triangle inequality, we get

$$
\begin{gathered}
p_{X} \leq q_{X} \leq h, \quad r_{X}^{\prime} \leq q_{X}^{\prime} \leq h, \quad s_{X} \leq h \quad(X \in \mathscr{F}), \\
r_{X} \leq h, \quad s_{X}^{\prime} \leq q_{X}, \quad t_{X} \leq q_{X}^{\prime} \quad(X \in \mathscr{B}) .
\end{gathered}
$$


As for $p$ and $q$, corresponding to types $q^{\prime}, r, r^{\prime}, s, s^{\prime}, t$ we obtain uniformities and set convergences. We remark that Hausdorff convergence is the finest among them. As in $\S 3$, for $\mathscr{S}(E) \subset \mathfrak{X}$ we get that $\mathfrak{X}(r)$ and $\mathfrak{X}\left(s^{\prime}\right)$ are finer than $W$ and so they are separated. If $\mathscr{A} \subset \mathfrak{X}$ then $(\mathscr{A}, \mathfrak{X}, s)$ is separated, too. Note that, generally, $\left(\mathscr{A}, \mathfrak{X}, r^{\prime}\right),(\mathscr{A}, \mathfrak{X}, t),\left(\mathscr{A}, \mathfrak{X}, q^{\prime}\right)$ are not separated, even for $E=\mathbf{R}^{2}, \mathscr{A}=\mathscr{H}(E) \cap \mathscr{C}(E), \mathscr{S}(E) \subset \mathfrak{X}$, as the following example (due to B. Le Gac) shows: take $\|(\xi, \eta)\|=|\xi|+|\eta|, A=\bar{B}(0,1)$, $B=[(-1,0),(1,0)]$; then $\rho(u, A)=\rho(u, B) \quad \forall u \in \mathbf{R}^{2}$.

Remark that the distal topology, introduced in [BP] for $E$ a n.v.s. and $\mathscr{A}=$ $\mathscr{C}(E), \mathfrak{X}=\mathscr{B}(E)$, is induced by $(\mathscr{A}, \mathfrak{X}, r)$. We also note that $\left[\mathscr{B}\left(s^{\prime}\right)\right.$ and $\mathscr{B}(s)] \Leftrightarrow H$ on $\mathscr{A}=\mathscr{B}(E), Z \Rightarrow \mathscr{B}(r)$ on $\mathscr{F}(E)$ (see Corollary 7.3.), $Z \Rightarrow \mathscr{B}(t)$ on $\mathscr{B}(E)$ (Corollary 7.3), Z $\Leftrightarrow \mathscr{F}(s)$ on $\mathscr{B}(E)$ (Theorem 7.2), $Z_{\rho} \Rightarrow \mathscr{B}(r)$ on $\mathscr{F}(E)$ (Corollary 9.3), $H_{\rho} \Rightarrow \mathscr{B}\left(s^{\prime}\right)$ on $\mathscr{F}(E)$, and if $A=$ $\mathscr{B}\left(s^{\prime}\right)-\lim \left(A_{i}\right)$ then $e_{\rho}\left(A, A_{i}\right)=0$.

We hope that some of these types will be useful in the study of set convergences. After the paper was submitted, several interesting results were obtained for the convergence induced by $(\mathscr{C} \cap \mathscr{B})(p)$ on $\mathscr{C}$ by $\mathrm{G}$. Beer in [Be7, 8]. He calls it the slice convergence. Being equivalent to Mosco convergence in reflexive Banach spaces, it seems that it is the most appropriate substitute for Mosco convergence in general normed spaces.

\section{REFERENCES}

[At] H. Attouch, Variational convergence for functions and operators, Pitman, Boston, Mass., and London, 1984.

[AAB] H. Attouch, D. Azé, and G. Beer, On some inverse stability problems for the epigraphical sum, Nonlinear Anal. TMA 16 (1991), 251-254.

[ALW] H. Attouch, R. Lucchetti, and R. Wets, The topology of the $\rho$-Hausdorff distance, Ann. Mat. Pura Appl. 160 (1991), 303-320.

[AW1] H. Attouch and R. Wets, Quantitative stability of variational systems. I. The epigraphical distance, Trans. Amer. Math. Soc. 328 (1991), 695-729.

[AW2] __, Quantitative stability of variational systems II. A framework for nonlinear conditioning, Working paper, IIASA, Laxemburg, Austria, 1988.

[AW3] _ Lipschitzian stability of the epsilon-approximate solutions in convex optimisation, Working paper, IIASA, Laxemburg, Austria, 1987.

[Az1] D. Azé, On some metric aspects of set-convergence, Preprint, AVAMAC, Univ. de Perpignan, 1988.

[Az2] _- Remarks on embeddings of hyperspaces into functional spaces, Preprint, AVAMAC, Univ. de Perpignan, 1989.

[AP1] D. Azé and J.-P. Penot, Operations on convergent families of sets and functions, Optimization 21 (1990), 521-534.

[AP2] _ Recent quantitative results about the convergence of convex sets and functions, Functional Anal. Approx., (P. L. Papini, ed.), Bagni di Lucca, Italy, 1988.

[BaP] M. Baronti and P. L. Papini, Convergence of sequences of sets, Methods of Funct. Anal. in Approx. Theory, Proc. Internat. Conf. (Bombay, 1985), Internat. Ser. of Numer. Math. 76, Birkhäuser, Basel, 1986.

[Be1] G. Beer, Metric spaces with nice closed balls and distance functions for closed sets, Bull. Austral. Math. Soc. 35 (1987), 81-96.

[Be2] _ On Mosco convergence of convex sets, Bull. Austral. Math. Soc. 38 (1988), 239-253.

[Be3] _ Support and distance functionals for convex sets, Numer. Funct. Anal. Optim. 10 (1989), 15-36. 
[Be4] _ Convergence of continuous linear functionals and their level sets, Arch. Math. 52 (1989), 482-489.

[Be5] _ Conjugate convex functions and the epi-distance topology, Proc. Amer. Math. Soc. 108 (1990), 117-126.

[Be6] _ A Polish topology for the closed subsets of a Polish space, Proc. Amer. Math. Soc. 113 (1991), 1123-1134.

[Be7] _ Topologies on closed and closed convex sets and the Effros measurability of set valued functions, Sém. Anal. Convexe, Montpellier, exposé no. 2, 1991.

[Be8] __ The slice topology a viable alternative to Mosco convergence in nonreflexive spaces, Sém. Anal. Convexe, Montpellier, exposé no. 3, 1991; Nonlinear Anal. TMA 19 (1992), 271-290.

[BBo] G. Beer and J. Borwein, Mosco convergence and reflexivity, Proc. Amer. Math. Soc. 109 (1990), 427-436.

[BHPVV] G. Beer, C. Himmelberg, C. Prikry, and F. Van Vleck, The locally finite topology on $2^{X}$, Proc. Amer. Math. Soc. 101 (1987), 168-172.

[BLLN] G. Beer, A. Lechicki, S. Levi, and S. Naimpally, Distance functionals and suprema of hyperspace topologies, Ann. Mat. Pura Appl. (to appear).

[BLu] G. Beer and R. Lucchetti, Weak topologies for the closed subspaces of a metrizable space, Trans. Amer. Math. Soc. 335 (1993), 805-822.

[BP] G. Beer and D. Pai, On convergence of convex sets and relative Chebyshev centers, J. Approx. Theory 62 (1990), 147-169.

[Ber] C. Berge, Espaces topologiques. Fonctions mutivoques, Dunod, Paris, 1959.

[BoFi] J. Borwein and S. Fitzpatrick, Mosco convergence and the Kadec property, Proc. Amer. Math. Soc. 106 (1989), 843-849.

[Bou1] N. Bourbaki, Topologie générale, Chapitres 1 et 2, Hermann, Paris, 1965.

[Bou2] _ , Topologie générale, Chapitre 9, Hermann, Paris, 1958.

[Bou3] _ Topologie générale, Chapitre 10, Hermann, Paris, 1961.

[CoPe] L. Contesse and J.-P. Penot, Continuity of polarity and conjugacy for the epi-distance topology, J. Math. Anal. Appl. (to appear).

[Co] B. Cornet, Topologies sur les fermés d'un espace métrique, Cahiers Math. Décision, ParisDauphine, 1974.

[DaK] Z. Daffer and H. Kaneko, Best approximation in metric spaces, Ann. Soc. Sci. Bruxelles Ser. I 96 (1982), 17-27.

[DBM] F. S. De Blasi and J. Myjak, Weak convergence of convex sets in Banach spaces, Arch. Math. 47 (1986), 448-456.

[DS] N. Dunford and J. T. Schwartz, Linear operators. I, Interscience, New York, 1967.

[Ef] E. G. Effros, Convergence of closed subsets in a topological space, Proc. Amer. Math. Soc. 16 (1965), 929-931.

[Fe] J. M. G. Fell, A Hausdorff topology for the closed subsets of a locally compact non-Hausdorff space, Proc. Amer. Math. Soc. 13 (1962), 472-476.

[Fi1] B. Fisher, A reformulation of the Hausdorff metric, Rostock. Math. Kolloq. 24 (1983), 7176.

[Fi2] _ Common fixed points of mappings and set-valued mappings, Rostock. Math. Kolloq. 18 (1981), 69-77.

[FiL] S. Fitzpatrick and A. S. Lewis, Weak* convergence of convex sets, Preprint, 1989.

[Fl] K. Floret, Weakly compact sets, Lecture Notes in Math., vol. 801, Springer-Verlag, Berlin, 1980.

[FrLL] S. Francaviglia, A. Lechicki, and S. Levi, Quasi-uniformization of hyperspaces and convergence of nets of semi-continuous multifunctions, J. Math. Anal. Appl. 112 (1985), 347-370.

[Fr] Z. Frólik, Concerning topological convergence of sets, Czechoslovak Math. J. 10 (85) (1960), 168-180. 
[He] C. Hess, Contribution à l'étude de la mesurabilité, de la loi de probabilité et de la convergence des multifonctions, Thèse d'Etat, U.S.T.L. Montpellier, 1986.

[Ho] R. Holmes, Geometrical functional analysis and its applications, Springer-Verlag, New York, 1975.

[Ke] J. L. Kelley, General topology, Van Nostrand, New York, 1955.

[KT] E. Klein and A. C. Thompson, Theory of correspondences, including applications to mathematical economics, Wiley, New York, 1984.

[Kö] G. Köthe, Topological vector spaces I, Springer-Verlag, Berlin, 1969.

[Kur] C. Kuratowski, Topologie, vol. I, Warsaw, Poland, 1958.

[LeL] A. Lechicki and S. Levi, Wijsman convergence in the hyperspace of a metric space, Bull. Un. Mat. Ital. (7) I-B (1987), 439-451.

[Lu1] R. Lucchetti, $A$ close look at the $A W$-topology, Conference Salerno, Italy, 1990.

[Lu2] _ Set convergences with applications to optimization and probability, Ph.D. thesis, Univ. of Calif., Davis, Calif., 1989.

[LSW] R. Lucchetti, G. Salinetti, and R. Wets, Uniform convergence of probability measures: topological criteria, Preprint, 1989.

[Ma] G. Matheron, Random sets and integral geometry, Wiley, New York, 1975.

[Mi] E. Michael, Topologies on spaces of subsets, Trans. Amer. Math. Soc. 71 (1951), 152-182.

[Mo1] U. Mosco, Convergence of solutions of variational inequalities, Adv. in Math. 3 (1969), 510-585.

[Mo2] _ - On the continuity of the Young-Fenchel transform, J. Math. Anal. Appl. 35 (1971), 518-535.

[Mr1] S. Mrówka, On the convergence of nets of sets, Fund. Math. 45 (1958), 237-246.

[Mr2] - Some comments on the spaces of subsets, Lecture Notes in Math., vol. 171, SpringerVerlag, 1970, pp. 59-63.

[Pe1] J-P. Penot, Preservation of stability and persistence under intersections and operations, Preprint, 1987.

[Pe2] _ Topologies and convergences on the space of convex functions, Nonlinear Anal. TMA 18 (10) (1992), 905-916.

[SaW] G. Salinetti and R. Wets, Convergence of convex sets in finite dimension, SIAM Rev. 21 (1979), 18-33.

[Sh] P. Shunmugaraj, On stability aspects in optimization, Ph.D. thesis, Indian Inst. Tech., Bombay, India, 1990.

[ShP] P. Shunmagaraj and D. Pai, On stability of approximate solutions of minimization problems, Preprint, Indian Inst. Tech. Bombay, India, 1990.

[So1] Y. Sonntag, Convergence au sens de U. Mosco; théorie et applications à l'approximation des solutions d'inéquations, Thèse d'Etat, Université de Provence, Marseille, 1982.

[So2] _ Convergence des suites d'ensembles, Monograph, Université de Provence, Marseille, 1987.

[SoZ1] Y. Sonntag and C. Zălinescu, Scalar convergence of sequences of convex sets, J. Math. Anal. Appl. 164 (1992), 219-241.

[SoZ2] _- Set convergences. An attempt of classification, Differential Equations and Control Theory (V. Barbu, ed.), Pitman Research Notes in Math., no. 250, 1991, pp. 312-323.

[Wi] R. A. Wijsman, Convergence of sequences of convex sets, cones and functions, Trans. Amer. Math. Soc. 123 (1966), 32-45.

[Ză] C. Zălinescu, Continuous dependence on data in abstract control problems, J. Optim. Theory Appl. 43 (1984), 277-306.

[Zo] T. Zolezzi, Approximazioni e perturbazioni di problemi di minimo, Book in preparation, Univ. of Genova, Italy.

Universite de Provence, Case X, 3 Place Victor Hugo, 13331 Marseille Cedex 3, FRANCE

Faculty of Mathematics, UNiversity Of IAȘI, 6600 IAși, România 